\title{
Abstract
}

ZACK, LAURIE. Nilpotent Lie Algebras with a Small Second Derived Quotient. (Under the direction of Ernie L. Stitzinger.)

There are many parallels between groups and Lie algebras, and mathematicians have been studying the similarities between them for decades. Many times researchers can look at results from group theory and translate them over into results in Lie algebras and vice versa. In 2003, Csaba Schneider published a paper in the Journal of Algebra about finite $p$-groups $G$, with the properties $G^{\prime \prime} \neq 1$ and $\left|G^{\prime} / G^{\prime \prime}\right|=p^{3}$. Schneider used Lie algebra calculations to inspire the ideas behind the group structure when $G$ is generated by two elements. He then extended the group ideas to find the structure of $G$ when generated by more than two elements and stated that it would be interesting to look at these results in Lie algebras. This paper completes the analogous Lie algebra problem, when $L$ is a nilpotent Lie algebra with properties $\operatorname{dim}\left(L^{\prime} / L^{\prime \prime}\right)=3$ and $L^{\prime \prime} \neq 0$. In this paper, not only have we found all the Lie algebra analogues to Schneider's results, we have also classified these algebras over the complex numbers. 


\section{NILPOTENT LIE ALGEBRAS WITH A SMALL SECOND DERIVED QUOTIENT}

by

\section{LAURIE MARGARET ZACK}

A dissertation submitted to the Graduate Faculty of North Carolina State University in partial fulfillment of the requirements for the Degree of Doctor of Philosophy

\section{MATHEMATICS}

Raleigh, North Carolina

2007

\section{APPROVED BY}

Dr. Robert Martin Committee Member

Dr. Kailash Misra

Committee Member
Dr. Tom Lada

Committee Member

Dr. Ernest Stitzinger

Chair of Advisory Committee 


\section{Biography}

Laurie Margaret Dolgas Zack was born December 23, 1979 in Grand Rapids, Michigan to James and Deborah Dolgas. She graduated Senior Class President at Rancho Bernardo High School in Rancho Bernardo, CA in 1998 before entering college at the University of Arkansas. During her four years at Arkansas, she swam for the Lady Razorbacks and worked in the Mathematics Multimedia Center. She graduated with a B.S. in Applied Mathematics with a minor in Education in 2002.

Upon graduation, she married Buffalo, NY native Eric Zack and then they moved to Raleigh, NC where she attended graduate school at North Carolina State University. She received her M.S. in Applied Mathematics in 2005 before switching to the theoretical side of math for her research and doctorate. She was also an adjunct faculty member at Meredith College from 2004-2007 teaching service level mathematics courses. 


\section{Acknowledgments}

Most importantly, I would like to thank my advisor and mentor Dr. Ernie Stitzinger. Without his help and patience, I would have never made it through my years in graduate school. He is like a second family to me, and I appreciate the countless hours of talking about everything and anything, his door was always open and I value all the opinions and advice he has given me. In addition, I would like to thank my parents, James and Deborah, for always supporting me and the decisions I have made in life, as well as my sister Krista, who I have looked to and received guidance for in the many obstacles I have encountered. Finally, to my husband and best friend, I want to give all my love and appreciation. He has followed me in the pursuit of my dreams and given me support along every step of the way. I cannot say Thank You enough. 


\section{Contents}

List of Tables $\quad$ v

1 Introduction $\quad 1$

2 Preliminaries $\quad 3$

3 A General Lemma and some Consequences 4

3.1 Proof of Theorem $1 \ldots \ldots \ldots$. . . . . . . . . . . . 7

3.2 Proof of Theorem $2 \ldots \ldots \ldots \ldots \ldots$

4 Central Decomposition Theorem $\quad 21$

5 The Structure of U $\quad 31$

6 Classification of $\mathbf{L}=\mathbf{H}+\mathbf{U}$ when $\mathbf{H}=\langle a, b\rangle \quad 32$

7 Isomorphism Classes when $\mathbf{H}=\langle a, b, u\rangle \quad 37$

8 Isomorphism Classes for $H=\left\langle a, b, u_{1}, u_{2}\right\rangle \quad 40$

9 Isomorphism Classes for $H=\left\langle a, b, u_{1}, u_{2}, u_{3}\right\rangle \quad 47$

10 Examples $\quad 54$

$\begin{array}{ll}\text { Bibliography } & 59\end{array}$ 


\section{List of Tables}

6.1 Two Generated Multiplication Table . . . . . . . . . . . . . . . . 33

6.2 Two Generated Multiplication Table Case 1 . . . . . . . . . . . . . . . . 35

6.3 Two Generated Multiplication Table Case 2 . . . . . . . . . . . . . . 35

7.1 Possible Isomorphisms . . . . . . . . . . . . . . . . . . . . . 38

8.1 Five Generated Possible Isomorphisms . . . . . . . . . . . . . . . . 44

9.1 Isomorphism Classes . . . . . . . . . . . . . . . . . . 52






\section{Chapter 1}

\section{Introduction}

In a recent paper, Csaba Schneider [5] found the structure of finite $p$-groups with properties $\left|G^{\prime} / G^{\prime \prime}\right|=p^{3}$ and $G^{\prime \prime} \neq 1$ for odd primes. He found that $\left|G^{2} / G^{3}\right|=p=$ $\left|G^{3} / G^{4}\right|=\left|G^{4} / G^{5}\right|$, and that $G^{5}=G^{\prime \prime}$. He went on to prove that $G$ is a central product of two simpler groups, $H$ and $U$, where $H$ is generated by at most five elements, $U^{\prime} \subseteq G^{5}$, and that $H$ and $U$ centralize each other. Schneider commented that to obtain the first of these results, he solved the analogous Lie algebra problem and then followed a similar pattern in $p$-groups. This paper was inspired by another one of Schneider's remarks that it would be interesting to solve the Lie algebra analog to the central product result. In this papaer, after obtaining this Lie algebra decomposition, $L=H+U$, we go on to classify the possible $H$ 's and $U$ 's over the complex numbers. We find $H$ can be one of fourteen isomorphism classes and $U$ is the direct sum of a generalized Heisenberg Lie algebra and an abelian Lie algebra.

For the analog to the second part of Schneider's results, we show general results which correspond to work of P. Hall and N. Blackburn [2], and in particular use the Lie algebra counterpart to a subgroup defined by Blackburn [1]. Finally, we find the fourteen possible classifications of $H$ over the complex numbers, results which as of yet 
have no group theory analog. 


\section{Chapter 2}

\section{Preliminaries}

We begin by introducing notation and definitions to be used throughout the remainder of the paper. The majority of our definitions come from notes written for a Lie algebra class at North Carolina State University [4]. Let $L$ be a finite dimensional nilpotent Lie algebra of class $t, L^{i}$ the $i$ th term of the lower central series, and we define the derived series $L^{\prime}=[L, L], L^{\prime \prime}=\left[L^{\prime}, L^{\prime}\right]$, and $L^{\prime \prime \prime}=\left[L^{\prime \prime}, L^{\prime \prime}\right]$. So there exists a chain $L \supseteq L^{1} \supseteq L^{2} \supseteq \cdots \supset L^{t+1}=0$ where $L^{2}=[L, L], L^{3}=\left[L, L^{2}\right], \ldots, L^{n}=\left[L, L^{n-1}\right]$ and $\mathrm{L}$ is said to be nilpotent of class $t$. Using notation from N. Blackburn [1], we will define $L^{1}=\left\{x \in L \mid\left[x, L^{2}\right] \subseteq L^{4}\right\}$. By definition, Lie algebras satisfy the Jacobi identity,

$$
[x,[y, z]]=[[x, y], z]+[y,[x, z]] \text { for all } x, y, z \in L
$$

which is heavily used throughout many of the proofs in this paper. Since many times we will be using more than three elements, we will simplify the bracket structure when the brackets are left justified we will use $\left[\cdots\left[\left[x_{1}, x_{2}\right], x_{3}\right], \cdots, x_{n}\right]=\left[x_{1}, x_{2}, \ldots, x_{n}\right]$. 


\section{Chapter 3}

\section{A General Lemma and some Consequences}

We start with a general lemma and some results that parallel Schneider's work.

Lemma 1. Let $L$ be a nilpotent Lie algebra and $H$ is a subalgebra of $L$ such that $L^{2}=$ $H^{2}+L^{3}$. Then

$$
\begin{gathered}
L^{i}=H^{i}+L^{i+1} \quad \text { for all } i \geq 2 \text { and } \\
L^{i}=H^{i} \quad \text { for all } i \geq 2 .
\end{gathered}
$$

Moreover, $H$ is an ideal of $L$.

Proof. We will use induction on $i$.

For $i=2$ we have $L^{2}=H^{2}+L^{3}$ which is the assumption of the lemma. Suppose that $L^{i-1}=H^{i-1}+L^{i}$ is true for $i-1 \geq 2$. Now we will show the result for $i$. We know that $H^{i}+L^{i+1} \subseteq L^{i}$ because $L^{i+1} \subseteq L^{i}$ and $H^{i} \subseteq L^{i}$. So we need only show that 
$L^{i} \subseteq H^{i}+L^{i+1}$. By definition we have

$$
\begin{aligned}
L^{i} & =\left[L^{i-1}, L\right] \\
& =\left[H^{i-1}+L^{i}, L\right] \\
& =\left[H^{i-1}, L\right]+\left[L^{i}, L\right] \\
& =\left[H^{i-1}, L\right]+L^{i+1} .
\end{aligned}
$$

So it is enough to show that $\left[H^{i-1}, L\right] \subseteq H^{i}+L^{i+1}$ Now $\left[H^{i-1}, L\right]=\left[H^{i-2}, H, L\right]$, and by the Jacobi identity we have $\left[H^{i-2}, H, L\right] \subseteq\left[L, H^{i-2}, H\right]+\left[H, L, H^{i-2}\right]$. Since $H^{i-2} \subseteq L^{i-2}$ and $\left[L, L^{i-2}\right]=L^{i-1}$ we have that

$$
\begin{aligned}
{\left[L, H^{i-2}, H\right] } & \subseteq\left[L^{i-1}, H\right] \\
& =\left[H^{i-1}+L^{i}, H\right] \\
& =\left[H^{i-1}, H\right]+\left[L^{i}, H\right] \\
& \subseteq H^{i}+L^{i+1} \\
\text { and }\left[H, L, H^{i-2}\right] & \subseteq\left[L^{2}, H^{i-2}\right] \\
& =\left[H^{2}+L^{3}, H^{i-2}\right] \\
& =\left[H^{2}, H^{i-2}\right]+\left[L^{3}, H^{i-2}\right] \\
& \subseteq H^{i}+L^{i+1}
\end{aligned}
$$

So $\left[H^{i-1}, L\right]=\left[H^{i-2}, H, L\right] \subseteq H^{i}+L^{i+1}$. Therefore, $L^{i}=H^{i}+L^{i+1}$ for all $i \geq 2$.

Now if $\mathrm{L}$ is nilpotent of class $\mathrm{c}$ then $L^{c+1}=H^{c+1}=0$. Suppose $L^{i+1}=H^{i+1}$ for some $i$ such that $3 \leq i \leq c+1$. Then by the previous result we have $L^{i}=H^{i}+L^{i+1}$ $=H^{i}+H^{i+1}=H^{i}$. Then by induction, $L^{i}=H^{i}$ for all $i \geq 2$. Moreover, since $[H, L] \subseteq[L, L] \subseteq L^{2}=H^{2} \subseteq H$ then $H$ is an ideal of $L$ 
From this lemma, we obtain the following corollary, which again parallels work done by Schneider.

Corollary 1. Let L be a Lie algebra. Then

(i) If $\operatorname{dim}\left(L^{2} / L^{3}\right)=1$, then $L$ has a 2 generator ideal $H$ such that $L^{i}=H^{i}$ for all $i \geq 2$.

(ii) If $\operatorname{dim}\left(L^{2} / L^{3}\right)=2$, then $L$ has a 3 generator ideal $H$ such that $L^{i}=H^{i}$ for all $i \geq 2$.

Proof (i). Suppose $L^{2}=[a, b]+L^{3}$ for some $a, b \in L$ and $H=\langle a, b\rangle$. Since $L^{2}=[a, b]+L^{3} \subseteq H^{2}+L^{3} \subseteq L^{2}$, we have $L^{2}=H^{2}+L^{3}$. So by the previous lemma we have $\mathrm{H}$ is an ideal of $\mathrm{L}$ and $L^{i}=H^{i}$ for all $i \geq 2$.

Proof (ii). Suppose $\operatorname{dim}\left(L^{2} / L^{3}\right)=2$. Then $\left\{[a, b]+L^{3},[c, d]+L^{3}\right\}$ is a basis for $L^{2} / L^{3}$ for some $a, b, c, d \in L$. Now select the subalgebra $\mathrm{H}$ in $\mathrm{L}$ as follows:

if $[a, c],[a, d],[b, c],[b, d] \in L^{3}$ then let $H=\langle a, b+c, d\rangle$. Then looking at $H^{2}$ we get $[a, b+c]=[a, b]+[a, c] \in[a, b]+L^{3}$. So $[a, b]=[a, b+c]-[a, c] \in H^{2}+L^{3}$ and $[b+c, d]=[b, d]+[c, d] \in[c, d]+L^{3}$. So $[c, d]=[b+c, d]-[b, d] \in H^{2}+L^{3}$.

We have $[a, b]+L^{3} \subseteq H^{2}+L^{3}$ and $[c, d]+L^{3} \subseteq H^{2}+L^{3}$ which implies $L^{2} \subseteq H^{2}+L^{3}$. Therefore $L^{2}=H^{2}+L^{3}$ and the result holds by Lemma 1 .

Without loss of generality, suppose that $[a, c] \notin L^{3}$. So $[a, c]=\alpha[a, b]+\beta[c, d]$ $\bmod L^{3}$ for some $\alpha, \beta$ where at least one of them is not 0 . Now, if $\alpha \neq 0$ then set $H=\langle a, c, d\rangle$. Then $\alpha[a, b]=[a, c]-\beta[c, d] \in H^{2}$. Hence $[a, b] \in H^{2}$, and since $[c, d] \in H^{2}$, $L^{2} \subseteq H^{2}+L^{3}$ and $L^{2}=H^{2}+L^{3}$.

Otherwise set $H=\langle a, b, c\rangle$. Then similarly we have $L^{2}=H^{2}+L^{3}$. In either case, by the previous lemma, $\mathrm{H}$ is an ideal of $\mathrm{L}$ and $L^{i}=H^{i}$ for all $i \geq 2$. 


\subsection{Proof of Theorem 1}

We now move to our first theorem of characterizing the Lie algebras. This theorem helps with the classification of the Lie algebras later by finding the dimension of the second derived quotient.

Theorem 1. Let $L$ be a nilpotent Lie algebra over a field of with characteristic not equal to $2, \operatorname{dim}\left(L^{\prime} / L^{\prime \prime}\right)=3$, and $L^{\prime \prime} \neq 0$. Then $\operatorname{dim}\left(L^{2} / L^{3}\right)=1$ and $L^{\prime \prime}=L^{5}$.

Proof. Suppose $L$ is finite dimensional such that $\operatorname{dim}\left(L^{\prime} / L^{\prime \prime}\right)=3$. If $\operatorname{dim}\left(L^{\prime} / L^{3}\right)=$ 1 , then $L^{\prime \prime}=\left[L^{\prime}, L^{\prime}\right] \subseteq L^{5}$ because

$$
\begin{aligned}
{\left[L^{\prime}, L^{\prime}\right] } & =\left[\langle a\rangle+L^{3},\langle a\rangle+L^{3}\right] \\
& =\langle[\alpha a+x, \beta a+y]\rangle \text { for all } x, y \in L^{3} \\
& =\langle\alpha[a, y]+\beta[x, a]+[x, y]\rangle \text { for all } x, y \in L^{3} \\
& \subseteq\left[\langle a\rangle, L^{3}\right]+\left[\langle a\rangle, L^{3}\right]+\left[L^{3}, L^{3}\right] \\
& =\left[\langle a\rangle+L^{3}, L^{3}\right] \\
& =\left[L^{\prime}, L^{3}\right] \subseteq L^{5} .
\end{aligned}
$$

Thus there exists a chain of ideals $L \supset L^{\prime}=L^{2} \supset L^{3} \supset L^{4} \supset L^{5} \supseteq L^{\prime \prime} \neq 0$. Hence, $L^{\prime \prime}=L^{5}$ by the counting of dimensions.

Now assume the $\operatorname{dim}\left(L^{\prime} / L^{3}\right)=2$. By the previous corollary, there exists a three generator ideal $H=\langle a, b, c\rangle$ of $\mathrm{L}$ such that $H^{i}=L^{i}$ for all $i \geq 2$. Therefore we may work with $H$ and for the remainder of the proof we will call it $L$. Since $L^{\prime \prime} \subseteq L^{4}$ and by counting the dimensions, we have that $L^{\prime \prime}=L^{4}$. We may also assume that $L$ is nilpotent of class 4 , hence $L^{5}=0$.

Now $L^{\prime} / L^{3}$ is abelian with dimension 2. So there exists $\alpha, \beta, \delta$ not all equal to 0 such 
that

$$
\alpha[a, b]+\beta[a, c]+\delta[b, c]=0 \bmod L^{3} .
$$

The three vectors are linearly dependent, so if $\alpha=\beta=0$ then $\delta \neq 0$ would imply that $[b, c]=0 \bmod L^{3}$. If $\alpha=0, \beta \neq 0$ then $\beta[a, c]+\delta[b, c]=0 \bmod L^{3}$. Then let $a^{\prime}=\beta a+\delta b, b^{\prime}=b$ and $c^{\prime}=c$. Then $\left\langle a^{\prime}, b^{\prime}, c^{\prime}\right\rangle$ is still a generating set because

$$
\left[\begin{array}{l}
a^{\prime} \\
b^{\prime} \\
c^{\prime}
\end{array}\right]=\left[\begin{array}{lll}
\beta & \delta & 0 \\
0 & 1 & 0 \\
0 & 0 & 1
\end{array}\right]\left[\begin{array}{l}
a \\
b \\
c
\end{array}\right]
$$

where $\beta \neq 0$, so the representation matrix is nonsingular. Furthermore $\left[a^{\prime}, c^{\prime}\right]=[\beta a+$ $\delta b, c] \in L^{3}$.

Similarly, if $\alpha \neq 0, \beta=0$, then replace $a^{\prime \prime}=-\alpha a+\delta c, b^{\prime \prime}=b, c^{\prime \prime}=c$ and again $\left\langle a^{\prime \prime}, b^{\prime \prime}, c^{\prime \prime}\right\rangle$ is a generating set. Then

$$
\left[a^{\prime \prime}, b^{\prime \prime}\right]=[-\alpha a+\delta c, b]=-\alpha[a, b]+\delta[c, b]=-\alpha[a, b]-\delta[b, c] \in L^{3}
$$

Finally, if $\alpha \neq 0, \beta \neq 0$ replace $\tilde{a}=(\beta / \alpha) a+(\delta / \alpha) b, \tilde{b}=b+(\beta / \alpha) c, \tilde{c}=c$. Similarly, $\langle\tilde{a}, \tilde{b}, \tilde{c}\rangle$ is still a generating set and

$$
\begin{aligned}
{[\tilde{a}, \tilde{b}] } & =[(\beta / \alpha) a+(\delta / \alpha) b, b+(\beta / \alpha) c] \\
& =(\beta / \alpha)[a, b]+\left(\beta^{2} / \alpha^{2}\right)[a, c]+\left(\delta \beta / \alpha^{2}\right)[b, c] \\
& =\left(\beta \alpha[a, b]+\beta^{2}[a, c]+\delta \beta[b, c]\right)\left(\frac{1}{\alpha^{2}}\right) \\
& =(\alpha[a, b]+\beta[a, c]+\delta[b, c])\left(\frac{\beta}{\alpha^{2}}\right) \\
& =0 \bmod L^{3} \\
& \in L^{3} .
\end{aligned}
$$


Therefore, we may assume without loss of generality that $L=\langle a, b, c\rangle, L^{\prime} / L^{3}=$ $\left\langle[a, b]+L^{3},[a, c]+L^{3}\right\rangle$ and $[b, c] \in L^{3}$. Hence $L^{\prime}=\langle[a, b],[a, c]\rangle+L^{3}$.

Since $[b, c] \in L^{3}$, then $[b, c, a] \in L^{4}$ and also the Jacobi identity $[a, b, c]+[b, c, a]+$ $[c, a, b]=0$ implies $[a, b, c]=-[b, c, a]-[c, a, b]$. Hence,

$$
[a, b, c]=[a, c, b] \bmod L^{4}
$$

Since $L^{\prime \prime} \neq 0$ and $L^{5}=0$ then

$$
\begin{aligned}
L^{\prime \prime} & =\left[L^{\prime}, L^{\prime}\right]=\langle[[a, b],[a, c]]\rangle \\
& =\langle[a, b, a, c]+[a,[a, b, c]]\rangle \\
& =\langle[a, b, a, c]-[a, b, c, a]\rangle \neq 0
\end{aligned}
$$

and

$$
\begin{gathered}
{[[a, c],[a, b]]=[[a, c, a], b]+[a,[a, c, b]]=[a, c, a, b]-[a, c, b, a] \neq 0 \quad \text { and }} \\
{[a, c, b, a]=[a, b, c, a] \quad \text { by }(3.1)}
\end{gathered}
$$

If $[a, b, a] \in L^{4}$ then $[a, b, a, c] \in L^{5}=0$. Similarly, if $[a, b, c] \in L^{4}$ then $[a, b, c, a] \in$ $L^{5}=0$. So either $[a, b, a]$ or $[a, b, c] \notin L^{4}$. Similarly, either $[a, c, a]$ or $[a, c, b] \notin L^{4}$. Now $[b, c] \in L^{3}$ which forces $[a, c, b]+[b, a, c] \in L^{4}$. Hence $[a, c, b] \in L^{4}$ if and only if $[a, b, c] \in L^{4}$ by (3.1). Hence either $[a, c, a] \notin L^{4}$ or $[a, b, c] \notin L^{4}$.

Now suppose $[a, b, c] \in L^{4}$. Then both $[a, c, a]$ and $[a, b, a] \notin L^{4}$ but they are in $L^{3}$. So we will change bases such that $[a, b, c] \notin L^{4}$. Since $\operatorname{dim}\left(L^{3} / L^{4}\right)=1$, then there exists 
$\alpha \neq 0$ such that

$$
[a, b, a]=\alpha[a, c, a] \bmod L^{4}
$$

So $\quad[a, b-\alpha c, a]=0 \bmod L^{4}$. Then let $b^{\prime}=b-\alpha c$

which implies $\left[a, b^{\prime}, a\right]=0 \quad \bmod L^{4}$

Also, $\left[b^{\prime}, c\right]=[b-\alpha c, c]=[b, c] \in L^{3}$ and $\left[a, b^{\prime}, a\right] \in L^{4}$. Then $\left\langle a, b^{\prime}, c\right\rangle$ is a generating set since

$$
\left[\begin{array}{l}
a \\
b^{\prime} \\
c
\end{array}\right]=\left[\begin{array}{ccc}
1 & 0 & 0 \\
0 & 1 & -\alpha \\
0 & 0 & 1
\end{array}\right]\left[\begin{array}{l}
a \\
b \\
c
\end{array}\right]
$$

and again the matrix representation is nonsingular. Dropping the prime we have $L=$ $\langle a, b, c\rangle,[b, c] \in L^{3}$ and $[a, b, a] \in L^{4}$. So $[a, b, c] \notin L^{4}$.

Thus we may assume that

$$
[a, b, a] \in L^{4},[a, b, c] \notin L^{4} \text { and }[b, c] \in L^{3}
$$

which implies that

$$
\begin{aligned}
{[a, b, c] } & =[a, c, b] \quad \bmod L^{4} \\
{[a, b, c] } & =[a, c, b]+x \quad \text { where } x \in L^{4} \\
{[a, b, c, a] } & =[a, c, b, a]+[x, a] \quad \text { where }[x, a] \in L^{5}=0
\end{aligned}
$$

Thus $[a, b, c, a]=[a, c, b, a] \neq 0$ since $[a, b, a] \in L^{4}$. Hence $a \notin C_{L}\left(L^{3}\right)$.

On the other hand, by the Jacobi identity $[a, b, a, b]+[[a, b],[a, b]]+[[b,[a, b]], a]=0$. 
Therefore, $[a, b, a, b]=-[b, a, b, a]=[a, b, b, a]$. Now,

$$
[a, b, a] \in L^{4} \text { so }[a, b, a, b] \in L^{5}=0 \text {, hence }[a, b, b, a]=0 \text {. }
$$

Now if $[a, b, b] \notin L^{4}$ then $L^{3}=\left\langle[a, b, b], L^{4}\right\rangle$ and we have $a \in C_{L}\left(L^{3}\right)$ which is a contradiction. Hence,

$$
[a, b, b] \in L^{4}
$$

If $[a, c, a] \notin L^{4}$ then there exists some $\alpha \neq 0$ such that $[a, c, a]=\alpha[a, c, b] \bmod L^{4}$ using (3.1) and (3.3). So let $a^{\prime}=a-\alpha b$, then

$$
\left[a^{\prime}, c, a^{\prime}\right]=[a-\alpha b, c, a-\alpha b]=[a, c, a-\alpha b]+[\alpha b, c, a-\alpha b] \in L^{4}+\left[L^{3}, L\right] \subset L^{4}
$$

Also,

$$
\left[a^{\prime}, b, a^{\prime}\right]=[a-\alpha b, b, a-\alpha b]=[a, b, a]-[a, b, \alpha b] \in L^{4} \text { by (3.4) and (3.5). }
$$

In this new generating set $\left\langle a^{\prime}, b, c\right\rangle$, and dropping the prime, we have $[b, c] \in L^{3}$ and $[a, b, a],[a, b, b],[a, c, a] \in L^{4}$.

Then

$$
\begin{aligned}
0 & =[a, b, a, c]+[[a, c],[a, b]]+[c,[a, b], a] \\
& =[a, b, a, c]+[[a, c], a, b]+[a,[[a, c], b]]+[c,[a, b], a] \\
& =0+0-[a, c, b, a]-[a, b, c, a] \\
& =-2[a, b, c, a]
\end{aligned}
$$

since $[a, b, a] \in L^{4},[a, c, a] \in L^{4}$ and $[a, c, b]=[a, b, c] \bmod L^{4}$. So $[a, b, c, a]=0$ which 
is a contradiction unless the characteristic of $\mathrm{L}$ is 2 , and we have $\operatorname{dim}\left(L^{\prime} / L^{3}\right)=1$.

\subsection{Proof of Theorem 2}

The next theorem is the Lie algebra analog to results of P. Hall and N. Blackbrun. In order to prove this theorem, we need some preliminary results that are analogous to those in Schneider's paper.

Lemma 2. Let $L$ be a nilpotent Lie algebra with $\operatorname{dim}\left(L^{\prime} / L^{\prime \prime}\right)=3$ over a field of characteristic not equal to 2. Then $L^{1}$ has codimension 1 in $L$.

Proof. Since every commutator is a linear combination of left justified commutators, $\left[L^{2}, L^{2}\right] \subseteq L^{4}$, and $L^{2} \subseteq L^{1}$ by definition of $L^{1}$. We may take $L^{4}=0$ and by Theorem 1 , $\operatorname{dim}\left(L^{2} / L^{3}\right)=\operatorname{dim}\left(L^{3} / L^{4}\right)=1$. Now let $L^{2}=\langle y\rangle+L^{3}$ and $L^{3}=\langle z\rangle+L^{4}$. Then $f(x)=$ $[x, y]=\alpha_{x} z$ has a one dimensional image. Therefore the kernel of $f$ has codimension 1 in $L$ and clearly $\operatorname{ker} f=L^{1}$.

We immediately obtain the following corollary.

Corollary 2. Let $L$ be a nilpotent Lie algebra with $\operatorname{dim}\left(L^{\prime} / L^{\prime \prime}\right)=3$ over a field of characteristic not equal to 2. Suppose that $\operatorname{dim}\left(L / L^{\prime}\right)=2$. Then $L$ has generators a and $b$ such that $L=\langle a\rangle+L^{1}$ and $L^{1}=\langle b\rangle+L^{2}$.

For the remainder of the paper, when working with a two generated algebra, we will take generators $a$ and $b$ as stated in Corollary 2. The following lemma and proof are also needed in the proof of our next theorem and they parallel the p-group case from Schneider's paper.

Lemma 3. Let $L$ be a 2-generated finite dimensional nilpotent Lie algebra such that $\operatorname{dim}\left(L^{\prime} / L^{\prime \prime}\right)=3, \operatorname{dim}\left(L^{\prime} / L^{3}\right)=1$, and $L^{\prime \prime} \neq 0$. Then generators $a$ and $b$ of $L$ can be chosen such that the following hold: 
(i) $L^{2}=\langle[a, b]\rangle+L^{3}$;

(ii) $L^{3}=\langle[a, b, a]\rangle+L^{4}$ and $[a, b, b] \in L^{4}$;

(iii) $L^{4}=\langle[a, b, a, a]\rangle+L^{5}$ and $[a, b, a, b] \in L^{5}$;

(iv) $L^{5}=\langle[a, b, a, a, b]\rangle+L^{6}$ and $[a, b, a, a, a] \in L^{6}$.

Proof. Assume $L=\langle a, b\rangle$ where $a$ and $b$ are chosen such that

$$
L=\langle a\rangle+L^{1},\left[L^{1}, L^{2}\right] \subseteq L^{4}, L^{1}=\langle b\rangle+L^{2}
$$

and we have

$$
L \supset L^{\prime}=L^{2} \supset L^{3} \supset L^{4} \supset L^{5}=L^{\prime \prime} \supset L^{6}
$$

Now,

$$
L^{2}=[L, L]=\left[L, L^{1}\right]=[\alpha a+\beta b+x, \gamma b+y]=\langle[a, b]\rangle+L^{3} \text { where } x, y \in L^{2}
$$

$$
\text { and } \begin{aligned}
L^{3} & =\left[L, L^{2}\right]=\langle[\alpha a+\beta b+x, \gamma[a, b]+y]\rangle \text { where } x \in L^{2} \text { and } y \in L^{3} \\
& =\langle\alpha \gamma[a,[a, b]]+\beta \gamma[b,[a, b]]+\alpha[a, y]+\beta[b, y]+\gamma[x,[a, b]]+[x, y]\rangle \\
& \in\langle[a,[a, b]]\rangle+\left[L^{1}, L^{2}\right]+L^{4}+L^{4}+L^{4}+L^{5} .
\end{aligned}
$$

So $L^{3}=\langle[a, b, a]\rangle+L^{4}$ and $[a, b, b] \in\left[L^{2}, L^{1}\right] \in L^{4}$. Also, Since $[a, b, a, b]=[[a, b, b], a]+$ $[[a, b],[a, b]] \in L^{5}+0$, it follows that

$$
\begin{aligned}
L^{4} & =\left[L, L^{3}\right]=[\alpha a+\beta b+x, \gamma[a, b, a]+z] \text { where } z \in L^{4}, x \in L^{2} \\
& \in\langle[a, b, a, a]\rangle+L^{5}+L^{5}+\ldots
\end{aligned}
$$

Therefore,

$$
L^{4}=\langle[a, b, a, a]\rangle+L^{5} \text { and }[a, b, a, b] \in L^{5} .
$$


So we have shown (i)-(iii).

For (iv) we can assume $L^{6}=0$. Now

$$
\begin{aligned}
L^{5} & =\left[L^{\prime}, L^{\prime}\right]=[[a, b]+x,[a, b, a]+y] \text { where } x \in \gamma_{3} \text { and } y \in L^{4} \\
& =[[a, b],[a, b, a]]+L^{6}
\end{aligned}
$$

But $[a, b, a, a, b]=[[a, b, a, b], a]+[[a, b, a],[a, b]]$ with $[a, b, a, b, a] \in L^{6}$

yields $L^{5}=\langle[a, b, a, a, b]\rangle$, since $L^{6}=0$.

In general, $L^{5}=\langle[a, b, a, a, b]\rangle+L^{6}$. We will make a change of basis such that all of the above hold and $[a, b, a, a, a]=0$.

Now

$$
[a, b, a, a, a]=\alpha[a, b, a, a, b] \text { implies }[a, b, a, a, a-\alpha b]=0
$$

So let $a^{\prime}=a-\alpha b$. Then

$$
\begin{aligned}
{\left[a^{\prime}, b, a^{\prime}, a^{\prime}, a^{\prime}\right]=} & {[a-\alpha b, b, a-\alpha b, a-\alpha b, a-\alpha b] } \\
= & {[a, b, a-\alpha b, a-\alpha b, a-\alpha b] } \\
= & {[a, b, a, a, a-\alpha b]+[a, b,-\alpha b, a, a-\alpha b]+[a, b, a,-\alpha b, a-\alpha b] } \\
& \quad+[a, b, \alpha b, \alpha b, a-\alpha b]
\end{aligned}
$$

Each of these is 0 because $[a, b, b] \in L^{4},[a, b, a, b] \in L^{5}$ and (3.6). Hence $\left[a^{\prime}, b, a^{\prime}, a^{\prime}, a^{\prime}\right]=0$. 
Now we will show the first three properties hold when $a$ is replaced by $a^{\prime}$.

$$
\begin{aligned}
{\left[a^{\prime}, b\right] } & =[a, b]-\alpha[b, b]=[a, b] \text { which implies } L^{2}=\left[a^{\prime}, b\right]+L^{3} \\
{\left[a^{\prime}, b, a^{\prime}\right] } & =[a, b, a]-[a, b, \alpha b]=[a, b, a]+x \text { where } x \in L^{4} \\
\text { so } L^{3} & =\left[a^{\prime}, b, a^{\prime}\right]+L^{4} \text { and }\left[b, a^{\prime}, b\right]=[b, a, b] \in L^{4}
\end{aligned}
$$

Now $\left[a^{\prime}, b, a^{\prime}, a^{\prime}\right]=[a, b, a, a]-[a, b, \alpha b, a]-[a, b, a, \alpha b]+[a, b, \alpha b, \alpha b]$ where the last three terms are in $L^{5}$, so $L^{4}=\left[a^{\prime}, b, a^{\prime}, a^{\prime}\right]+L^{5}$. Also $\left[a^{\prime}, b, b\right]=[a, b, b] \in L^{4}$ and $\left[a^{\prime}, b, a^{\prime}, b\right]=[a, b, a, b]-[a, b, \alpha b, b] \in L^{5}$. Finally,

$$
\left[a^{\prime}, b, a^{\prime}, a^{\prime}, b\right]=[a, b, a, a, b]-[a, b, \alpha b, a, b]-[a, b, a, \alpha b, b]+[a, b, \alpha b, \alpha b, b]
$$

where the last three terms are in $L^{6}$ which implies $L^{5}=\left[a^{\prime}, b, a^{\prime}, a^{\prime}, b\right]$ since $L^{6}=0$.

So by letting $a=a^{\prime}$ we obtain the lemma.

The following is analogous to a lemma by Blackburn [2].

Lemma 4. If $L$ is a nilpotent Lie algebra over a field of characteristic not equal to 2, with series $L \supset L^{\prime} \supset L^{\prime \prime} \supset L^{\prime \prime \prime}=0$ where $\operatorname{dim}\left(L^{\prime} / L^{\prime \prime}\right)=3$ and $\operatorname{dim}\left(L^{\prime \prime}\right)=2$. Then the following hold:

(i) $L^{\prime \prime}=Z\left(L^{\prime}\right)$;

(ii) $L^{\prime}$ has a unique abelian subalgebra $A$ of codimension 1 in $L^{\prime}$;

(iii) If $B$ is an ideal of $L$ where $\operatorname{dim}(B)=3$ and $L^{\prime} \supset B \supset L^{\prime \prime}$, then $B \subset A$.

Proof. By Theorem $1, \operatorname{dim}\left(L^{2} / L^{3}\right)=1$. By Corollary 1 , there is a two generated ideal $H$ such that $L^{i}=H^{i}$ for all $i \geq 2$. The assumptions and assertions change over to $H$, so we can assume that $L$ is also two generated. Then 


$$
\begin{gathered}
L=\langle a, b\rangle \\
L=\langle a\rangle+L^{1} \\
L^{1}=\langle b\rangle+L^{2} \\
L^{2}=\langle[a, b]\rangle+L^{3} \\
L^{3}=\langle[a, b, a]\rangle+L^{4} \text { and }[a, b, b] \in L^{4} \\
L^{4}=\langle[a, b, a, a]\rangle+L^{5} \quad \text { and } \quad[a, b, a, b] \in L^{5} \\
L^{5}=\langle[a, b, a, a, b]\rangle+L^{6} \quad \text { and } \quad[a, b, a, a, a] \in L^{6}
\end{gathered}
$$

and $L \supset L^{1} \supset L^{2}=L^{\prime} \supset L^{3} \supset L^{4} \supset L^{5}=L^{\prime \prime} \supset L^{6} \supset L^{7}=0$.

Also, pick $k \in L^{6}$ such that $\{[a, b],[a, b, a],[a, b, a, a],[a, b, a, a, b], k\}$ is a basis for $L^{\prime}$.

(i). To show $L^{\prime \prime} \subseteq Z\left(L^{\prime}\right)$, first note that

$$
\left[L^{\prime \prime}, L^{\prime}\right]=\left[L^{5}, L^{2}\right] \subseteq L^{7}=0, \text { so } L^{\prime \prime} \subseteq Z\left(L^{\prime}\right)
$$

Now to show $Z\left(L^{\prime}\right) \subseteq L^{\prime \prime}$, suppose $L^{\prime \prime} \neq Z\left(L^{\prime}\right)$. Since $L^{\prime}$ is not abelian, $Z\left(L^{\prime}\right) \neq L^{\prime}$ and $Z\left(L^{\prime}\right)$ can never have codimension 1 in $L^{\prime}$ if $\operatorname{dim}\left(L^{\prime}\right)>1$. We claim that the dimension of $Z\left(L^{\prime}\right)=2$. If the dimension is 5 , then $L^{\prime}$ is abelian, which implies that $L^{\prime \prime}=0$, which is a contradiction. If the dimension of $Z\left(L^{\prime}\right)=4$, then $Z\left(L^{\prime}\right)$ has codimension 1 in $L^{\prime}$, which is a contradiction. If $\operatorname{dim}\left(Z\left(L^{\prime}\right)\right)=3$, let $\{s, t, z\}$ be a basis for $Z\left(L^{\prime}\right)$ which can be extended to a basis $\{x, y, s, t, z\}$ for $L^{\prime}$ since $L^{\prime}$ is 5 -dimensional. 
Then

$$
\begin{gathered}
{[x, s]=[x, t]=[x, z]=0} \\
{[y, s]=[y, t]=[y, z]=0} \\
{[s, t]=[s, z]=[t, z]=0} \\
\text { since } s, t, z \in Z\left(L^{\prime}\right) .
\end{gathered}
$$

Then $\left[L^{\prime}, L^{\prime}\right]=\langle[x, y]\rangle$ would imply $L^{\prime \prime}$ is 1 -dimensional which is a contradiction. Hence, $\operatorname{dim}\left(Z\left(L^{\prime}\right)\right)=2$ and $Z\left(L^{\prime}\right)=L^{\prime \prime}$. Since $L^{\prime} \supset L^{\prime \prime}=Z\left(L^{\prime}\right) \supset\left[L^{\prime}, L^{\prime \prime}\right]=0$ it follows that $L^{\prime}$ has class 2 .

(ii). Let $A=\langle[a, b, a],[a, b, a, a], s, t\rangle$ where $s, t \in L^{\prime \prime}$, and the vectors in the generating set form a basis for $A$. Then $\operatorname{dim}\left(L^{\prime}\right)=4$. Let $j=s$ or $t$. Then

$$
\begin{aligned}
& {[[a, b, a],[a, b, a, a]] \in L^{7}=0,} \\
& {[[a, b, a], j] \in L^{8}=0,} \\
& {[[a, b, a, a], j] \in L^{9}=0 .}
\end{aligned}
$$

Hence, $A$ is an abelian subalgebra of codimension 1 in $L^{\prime}$.

Now suppose there exists another 4-dimensional abelian subalgebra $B$ such that $B \subset L^{\prime}$. Then $A+B=L^{\prime}$ and $\operatorname{dim}(A \cap B)=3$. Hence let $\{s, t, z\}$ be a basis for $A \cap B$, so $A \cap B=\langle s, t, z\rangle$ where $s, t \in L^{5}=L^{\prime \prime}=Z\left(L^{\prime}\right)$. Also, let $\{x, z, s, t\}$ a basis for $A$ and $\{y, z, s, t\}$ a basis for $B$. Then $L^{\prime}=\langle x, y, z, s, t\rangle, L^{\prime \prime}=\left[L^{\prime}, L^{\prime}\right]=\langle[x, y]\rangle$ since $A$ and $B$ are abelian. Therefore $L^{\prime \prime}$ is 1-dimensional which is a contradiction. So $\mathrm{A}$ is unique.

(iii). Let $B$ be an ideal of $L, \operatorname{dim}(B)=3$ and $L^{\prime} \supset B \supset L^{\prime \prime}$. We will show that $B \subseteq A$. Let $x=[a, b], y=[a, b, a], z=[a, b, a, a]$ and $B=\langle w\rangle+L^{\prime \prime}$ where $w=\alpha[a, b]+\beta[a, b, a]+\delta[a, b, a, a]$. If $B \nsubseteq A$ then $B$ is a maximal abelian subalgebra of 
$L^{\prime}$. Now, $[w, a]=\alpha[a, b, a]+\beta[a, b, a, a]+\delta[a, b, a, a, a]$. Since $B$ is an ideal of $L$ we have $[w, a] \in\langle w\rangle+L^{5}$ so $[w, a]=c(\alpha[a, b]+\beta[a, b, a]+\delta[a, b, a, a])+k$ where $k \in L^{5}=L^{\prime \prime}$.

From the last two expressions, we have

$$
\alpha[a, b, a]+\beta[a, b, a, a]+\delta[a, b, a, a, a]=c(\alpha[a, b]+\beta[a, b, a]+\delta[a, b, a, a])+k
$$

Then $c \alpha=0, c \beta=\alpha$ and $c \delta=\beta$. Hence either $c=0$ or $\alpha=0$. If $c \neq 0$, then $\alpha=0$ implies $\beta=0$ implies $\delta=0$, which implies $w=0$ and $\operatorname{dim}(B)=2$, which is a contradiction. So then $c=0$, which implies that $\alpha=0$ and $\beta=0$. Therefore $B=\langle[a, b, a, a]\rangle+L^{5} \subseteq\langle[a, b, a],[a, b, a, a]\rangle+L^{5}=A$. Thus $B \subseteq A$.

Using the above lemmas and corollaries, we are able to prove our next theorem, which is analogous to work done by Blackburn and Hall [2].

Theorem 2. Suppose $L$ is a nilpotent Lie algebra of characteristic not 2 such that $\operatorname{dim}\left(L^{\prime} / L^{\prime \prime}\right)=3$ and $\operatorname{dim}\left(L^{\prime \prime}\right) \geq 1$. Then $\operatorname{dim}\left(L^{\prime \prime}\right)=1$.

\section{Proof.}

First assume all of the conditions in Lemma 4 hold. Then continuing with the notation as before, Let $x=[a, b], y=[a, b, a], z=[a, b, a, a], s=[x, y]$ and $r=[x, z]$. Then $[y, z] \in\left[L^{3}, L^{4}\right]=L^{7}=0$. Hence $\{r, s\}$ is a basis for $L^{5}=L^{\prime \prime}$.

We claim that $[x, g, y]=0$ for all $g \in L$. Now if $g=a$ then $[x, g, y]=[[a, b, a],[a, b, a]]$ $=0$. If $g=b$, we get $[x, g, y]=[[a, b, b],[a, b, a]] \subseteq\left[L^{4}, L^{3}\right]=L^{7}=0$. Thus

$$
[x, g, y]=0
$$

For $g \in L$ we have

$$
[r, g]=[x, z, g]=[x, g, z]+[x,[z, g]] \in L^{7}=0 .
$$


Hence $r \in Z(L)$.

Since $[y, g] \in L^{4}$, it follows that $[y, g]=\alpha z+u$ where $u \in L^{5}$. Then $[s, g]=[x, y, g]=$ $[x, g, y]+[x,[y, g]]=0+[x, \alpha z+u]=\alpha r$ since $u \in L^{5}=Z\left(L^{\prime}\right)$. Hence

$$
[s, g]=\alpha r \text { for any } g \in L
$$

Now $L^{5}=\langle s\rangle+L^{6}$ and $r$, which is the product of 6 terms, is in $L^{6}$, hence $L^{6}=\langle r\rangle$.

Letting $\left[y, g_{i}\right]=\alpha_{i} z \bmod L^{5}$. Then $\left[s, g_{i}\right]=\left[x, y, g_{i}\right]=\left[x, g_{i}, y\right]+\left[x,\left[y, g_{i}\right]\right]=$ $0+\left[x, \alpha_{i} z\right]=\alpha_{i} r$. Since $\{r, s\}$ is a basis for $L^{5}$ we have $\left[z, g_{i}\right]=\beta_{i} r+\epsilon_{i} s$.

Now

$$
\begin{array}{rlr}
{\left[z,\left[g_{1}, g_{2}\right]\right]} & =\left[z, g_{1}, g_{2}\right]+\left[g_{1},\left[z, g_{2}\right]\right] & \\
& =\left[\beta_{1} s+\xi_{1} r, g_{2}\right]+\left[g_{1}, \beta_{2} s+\xi_{2} r\right] & \\
& =\beta_{1} \alpha_{2} r+0-\alpha_{1} \beta_{2} r+0 & \\
& =-\left(\alpha_{1} \beta_{2}-\alpha_{2} \beta_{1}\right) r &
\end{array}
$$

and

$$
\begin{aligned}
{\left[y,\left[g_{1}, g_{2}\right]\right] } & =\left[y, g_{1}, g_{2}\right]+\left[g_{1},\left[y, g_{2}\right]\right] \\
& =\left[\alpha_{1} z, g_{2}\right]+\left[g_{2}, \alpha_{2} z\right] \\
& =\alpha_{1} \beta_{2} s+\alpha_{1} \epsilon_{2} r-\alpha_{2} \beta_{1} s-\alpha_{2} \epsilon_{1} r .
\end{aligned}
$$


Now let $g_{1}=a$ and $g_{2}=b$. Then

$$
\begin{aligned}
{\left[y,\left[g_{1}, g_{2}\right]\right] } & =[y,[a, b]]=-s \\
\left(\alpha_{1} \beta_{2}-\alpha_{2} \beta_{1}\right) s & =-s
\end{aligned}
$$

which implies $\alpha_{1} \beta_{2}-\alpha_{2} \beta_{1}=-1$

$$
\begin{aligned}
\text { and }\left[z,\left[g_{1}, g_{2}\right]\right] & =[z,[a, b]]=[z, x]=-r \\
-\left(\alpha_{1} \beta_{2}-\alpha_{2} \beta_{1}\right) r & =-r
\end{aligned}
$$

which implies $\alpha_{1} \beta_{2}-\alpha_{2} \beta_{1}=1$.

So $1=-1$ which is a contradiction unless the characteristic is 2 . Therefore, $\operatorname{dim}\left(L^{\prime \prime}\right)=1$.

Now suppose $\operatorname{dim}\left(L^{\prime \prime}\right)>2$. Then $L^{\prime \prime}=L^{5} \supset L^{6} \supset L^{7} \supset L^{\prime \prime \prime}$ and there exists a vector space $V$ such that $L^{6} \supset V \supseteq L^{7}$, and $\operatorname{dim}\left(L^{6} / V\right)=1$. Now $[L, V] \subseteq\left[L, L^{6}\right]=$ $L^{7} \subseteq V$. Thus $V$ is an ideal of $L$, so $L / V$ satisfies the assumptions of Lemma 4 , and $\operatorname{dim}\left((L / V)^{\prime \prime}\right)=2$, which we have just shown is not possible. Hence $L^{\prime \prime \prime}=0$ and $\operatorname{dim}\left(L^{\prime \prime}\right)=1$. 


\section{Chapter 4}

\section{Central Decomposition Theorem}

As we have already seen, $L$ has a 2-generator ideal $H$, such that for all $i \geq 2, L^{i}=H^{i}$. We have also found in the previous chapter, a particular generating set which satisfies some additional conditions. These results are used to obtain the desired factorization. This work again parallels Schneider's. Before the theorem, we need one additional lemma.

Lemma 5. Let $L$ be nilpotent Lie algebra over a field of characteristic not equal to 2, such that $\operatorname{dim}\left(L^{\prime} / L^{\prime \prime}\right)=3$ and $L^{\prime \prime} \neq 0$. Then $L$ has a minimal generating set $\left\{a, b, u_{1}, u_{2}, \ldots, u_{r}\right\}$ which is a basis for $L / L^{\prime}$ such that for all $u_{i}$ and $u_{j}$ :

(i) $H=\langle a, b\rangle$ is an ideal of $L$ such that $H^{i}=L^{i}$ for all $i \geq 2$. Furthermore, $a$ and $b$ are as in Lemma 4;

(ii) $\left[a, u_{i}\right] \in L^{5}$ for all $u_{i}$;

(iii) $\left[b, u_{i}\right] \in L^{4}$ for all $u_{i}$;

(iv) $\left[u_{i}, u_{j}\right] \in L^{5}$ for all $u_{i}$ and $u_{j}$.

Proof. From Theorem 1, under these conditions, we have $\operatorname{dim}\left(L^{\prime} / L^{3}\right)=1$, and from Corollary 1, $L$ has 2-generator ideal $H$ such that $L^{i}=H^{i}$ for $i \geq 2$. Then from Theorem 
2, we know $\operatorname{dim}\left(L^{\prime \prime}\right)=1, L^{\prime \prime}=L^{5}$, and $L^{6}=0$. So we will select $a, b \in L$ such that $H=\langle a, b\rangle$, where $a$ and $b$ are as in Lemma 3.

Now choose $u_{1}, u_{2}, \ldots, u_{r}$ such that $\left\{a, b, u_{1}, \ldots, u_{r}\right\}$ is a basis for a subspace complementary to $L^{2}$.

Suppose $\left[u_{i}, a\right]=\alpha_{i}[b, a] \bmod L^{3}$ and $\left[u_{i}, b\right]=\beta_{i}[b, a] \bmod L^{3}$. Then

$$
\begin{aligned}
{\left[u_{i}-\alpha_{i} b+\beta_{i} a, b\right] } & =\left[u_{i}, b\right]-\alpha_{i}[b, b]+\beta_{i}[a, b] \\
& =\left[u_{i}, b\right]+\beta_{i}[a, b] \\
& =\beta_{i}[b, a]+\beta_{i}[a, b] \bmod L^{3} \\
& \in L^{3} .
\end{aligned}
$$

Also, $\left[u_{i}-\alpha_{i} b+\beta_{i} a, a\right]=\left[u_{i}, a\right]-\alpha_{i}[b, a]+\beta_{i}[a, a]$

$$
\begin{aligned}
& =\left[u_{i}, a\right]+\alpha_{i}[b, a] \\
& =\alpha_{i}[b, a]-\alpha_{i}[b, a] \bmod L^{3} \\
& \in L^{3} .
\end{aligned}
$$

So, let $u_{i}^{\prime}=u_{i}-\alpha_{i} b+\beta_{i} a$. Then $\left\{a, b, u_{1}^{\prime}, u_{2}^{\prime}, \ldots, u_{r}^{\prime}\right\}$ is also a basis complementary to $L^{2}$ since

$$
\left[\begin{array}{c}
a \\
b \\
u_{1}^{\prime} \\
u_{2}^{\prime} \\
\vdots \\
u_{r}^{\prime}
\end{array}\right]=\left[\begin{array}{cccccc}
1 & 0 & 0 & 0 & \cdots & 0 \\
0 & 1 & 0 & 0 & \cdots & 0 \\
\beta_{1} & -\alpha_{1} & 1 & 0 & \cdots & 0 \\
\beta_{2} & -\alpha_{2} & 0 & 1 & \cdots & 0 \\
\vdots & \vdots & \vdots & & \ddots & \vdots \\
\beta_{r} & -\alpha_{r} & 0 & 0 & \cdots & 1
\end{array}\right]\left[\begin{array}{c}
a \\
b \\
u_{1} \\
u_{2} \\
\vdots \\
u_{r}
\end{array}\right]
$$

and the change of basis matrix is nonsingular. We also have $\left[a, u_{i}^{\prime}\right],\left[b, u_{i}^{\prime}\right] \in L^{3}$ for $i=$ $1,2, \ldots, r$. 
Suppose $\left[u_{i}^{\prime}, a\right]=\alpha_{i}[a, b, a]+\beta_{i}[a, b, a, a] \bmod L^{5}$. So $\left[u_{i}^{\prime}, a\right]-\alpha_{i}[a, b, a]-\beta_{i}[a, b, a, a]$ $=0 \bmod L^{5}$. Then let $u_{i}^{\prime \prime}=u_{i}^{\prime}-\alpha_{i}[a, b]-\beta_{i}[a, b, a]$. This implies $\left[u_{i}^{\prime \prime}, a\right] \in L^{5}$ and similarly $\left\{a, b, u_{1}^{\prime \prime}, u_{2}^{\prime \prime}, \ldots, u_{r}^{\prime \prime}\right\}$ is still a basis for the complementary subspace because the change of basis matrix is nonsingular. We claim $\left[u_{i}^{\prime \prime}, b\right] \in L^{4}$.

Now, $\left[a, b, u_{i}^{\prime \prime}\right] \in L^{4}$ since $\left[a, b, u_{i}^{\prime \prime}\right]=\left[a, u_{i}^{\prime \prime}, b\right]+\left[a,\left[b, u_{i}^{\prime \prime}\right]\right]$ by the Jacobi identity, and $\left[a, u_{i}^{\prime \prime}\right] \in L^{5}$, therefore $\left[a, u_{i}^{\prime \prime}, b\right] \in L^{6}=0$ and $\left[b, u_{i}^{\prime \prime}\right] \in L^{3}$ so $\left[a,\left[b, u_{i}^{\prime \prime}\right]\right] \in L^{4}$.

So we have the following:

$$
\begin{gathered}
{\left[u_{i}, a\right],\left[u_{i}, b\right] \in L^{2},} \\
{\left[u_{i}^{\prime}, a\right],\left[u_{i}^{\prime}, b\right] \in L^{3},} \\
{\left[u_{i}^{\prime \prime}, a\right] \in L^{5} \text {, and }\left[a, b, u_{i}^{\prime \prime}\right] \in L^{4} .}
\end{gathered}
$$

Hence,

$$
\left[a, b, a, u_{i}^{\prime \prime}\right]=\left[a, b, u_{i}^{\prime \prime}, a\right]+\left[[a, b],\left[a, u_{i}^{\prime \prime}\right]\right]=0
$$

since $\left[a, b, u_{i}^{\prime \prime}\right] \in L^{4}$, so $\left[a, b, a, u_{i}^{\prime \prime}, a\right] \in L^{6}=0$ and $\left[a, u_{i}^{\prime \prime}\right] \in L^{5}$ so $\left[[a, b],\left[a, u_{i}^{\prime \prime}\right]\right] \in L^{6}=0$.

Similarly,

$$
\left[a, b, a, a, u_{i}^{\prime \prime}\right]=\left[a, b, a, u_{i}^{\prime \prime}, a\right]+\left[[a, b, a],\left[a, u_{i}^{\prime \prime}\right]\right]=[0, a]+\left[L^{3}, L^{5}\right]=0 .
$$

Therefore $\left[u_{i}^{\prime \prime}, L^{3}\right]=0$.

Since $\left[u_{i}^{\prime \prime}, b\right] \in L^{3}$, then

$$
\left[u_{i}^{\prime \prime}, b\right]=\epsilon_{i}[a, b, a]+\delta_{i}[a, b, a, a] \bmod L^{5} .
$$


Since we want $\left[u_{i}^{\prime \prime}, b\right] \in L^{4}$, we want $\epsilon_{i}=0$. Now,

$$
\begin{aligned}
{\left[a, b, u_{i}^{\prime \prime}\right] } & =\left[a,\left[b, u_{i}^{\prime \prime}\right]\right]-\left[b,\left[a, u_{i}^{\prime \prime}\right]\right]=\left[u_{i}^{\prime \prime}, b, a\right] \text { since }\left[b,\left[a, u_{i}^{\prime \prime}\right]\right] \in L^{6}=0 \\
& =\epsilon_{i}[a, b, a, a], \\
\text { so }\left[a, b, u_{i}^{\prime \prime}, b\right] & =\epsilon_{i}[a, b, a, a, b] . \\
\text { Now }\left[a, b, u_{i}^{\prime \prime}, b\right] & =\left[a, b, b, u_{i}^{\prime \prime}\right]+\left[[a, b],\left[u_{i}^{\prime \prime}, b\right]\right] \text { by the Jacobi identity. } \\
& =\left[a, b,\left[u_{i}^{\prime \prime}, b\right]\right] \text { since }[a, b, b] \in L^{4} \text { and }\left[u_{i}^{\prime \prime}, L^{4}\right]=0 . \\
& =\left[[a, b], \epsilon_{i}[a, b, a]\right] \\
& =\left[a, \epsilon_{i}[a, b, a], b\right]+\left[a,\left[b, \epsilon_{i}[a, b, a]\right]\right] \text { by the Jacobi identity. } \\
& =-\epsilon_{i}[a, b, a, a, b]+0 \\
& \text { since }\left[b, \epsilon_{i}[a, b, a]\right] \in L^{5} \text { so }\left[a,\left[b, \epsilon_{i}[a, b, a]\right]\right] \in L^{6}=0 .
\end{aligned}
$$

Therefore, $\epsilon_{i}[a, b, a, a, b]=-\epsilon_{i}[a, b, a, a, b]$, and $[a, b, a, a, b] \neq 0$ since it is a basis element, hence $\epsilon_{i}=0$ and $\left[u_{i}^{\prime \prime}, b\right] \in L^{4}$ by $(4.3)$.

Now we will drop the primes for simplicity and show that $u_{1}, u_{2}, \ldots, u_{r} \in C\left(L^{\prime}\right)$. We know $L^{\prime}=L^{2}=\langle[a, b],[a, b, a],[a, b, a, a],[a, b, a, a, b]\rangle$. We already have that $\left[u_{i},[a, b, a]\right]$ $=\left[a, b, a, u_{i}\right]=0$, and $\left[a, b, a, a, u_{i}\right]=0$. Clearly, $\left[a, b, a, a, b, u_{i}\right] \in L^{6}=0$, so we just need to show that $\left[u_{i},[a, b]\right]=0$. Consider

$$
\left[u_{i},[a, b]\right]=\left[u_{i}, a, b\right]+\left[a,\left[u_{i}, b\right]\right]=0
$$

Since $\left[u_{i}, b\right] \in L^{4}$, then $\left[a,\left[u_{i}, b\right]\right] \in L^{6}=0$. We also have $\left[u_{i}, a\right] \in L^{5}$ and $\left[u_{i}, a, b\right]=0$. Therefore the $u_{i}$ 's centralize $L^{\prime}$.

It remains to show $\left[u_{i}, u_{j}\right] \in L^{5}$. We know that $\left[u_{i}, u_{j}\right] \in L^{\prime}=L^{2}=H^{2}=H^{\prime}$, and 
for all $k=1,2, \ldots, r$ we have

$$
\left[\left[u_{i}, u_{j}\right], a\right]=\left[u_{i},\left[u_{j}, a\right]\right]+\left[\left[u_{i}, a\right], u_{j}\right]=0 \text { since }\left[u_{k}, a\right] \in L^{5},\left[u_{k},\left[u_{k}, a\right]\right] \in L^{6}=0 .
$$

Also, $\left[\left[u_{i}, u_{j}\right], b\right]=\left[u_{i},\left[u_{j}, b\right]\right]+\left[\left[u_{i}, b\right], u_{j}\right]=0$ since $\left[u_{k}, b\right] \in L^{4}$ and $u_{k}$ takes $L^{4}$ to 0 . Therefore $\left[\left[u_{i}, u_{j}\right],[a, b]\right]=0$ and $\left[u_{i}, u_{j}\right] \in Z(H)$ which implies $\left[u_{i}, u_{j}\right] \in Z(H) \cap H^{\prime}$, since $H^{\prime}=L^{\prime}$. Now, $H^{5} \subseteq H^{\prime} \cap Z(H)$. If $x \in H^{\prime}$ then

$$
x=c_{1}[a, b]+c_{2}[a, b, a]+c_{3}[a, b, a, a]+c_{4}[a, b, a, a, b] .
$$

If $x \in Z(H)$ then $[x, a]=0$. So we have

$$
\begin{aligned}
0=[x, a] & =c_{1}[a, b, a]+c_{2}[a, b, a, a]+c_{3}[a, b, a, a, a]+c_{4}[a, b, a, a, b, a] \\
& =c_{1}[a, b, a]+c_{2}[a, b, a, a], \text { since }[a, b, a, a, a] \in L^{6}=0
\end{aligned}
$$

So $c_{1}=0$, and $c_{2}=0$. Therefore $x=c_{3}[a, b, a, a]+c_{4}[a, b, a, a, b]$. Now $[x, b]=0$ and

$$
\begin{aligned}
0=[x, b] & =c_{3}[a, b, a, a, b]+c_{4}[a, b, a, a, b, b] \\
& =c_{3}[a, b, a, a, b]+0 .
\end{aligned}
$$

So $c_{3}=0$ and $x=c_{4}[a, b, a, a, b] \in L^{5}$. Therefore $H^{\prime} \cap Z(H)=H^{5}=L^{5}$, hence $\left[u_{i}, u_{j}\right] \in L^{5}$.

Now we are able to prove the central decomposition theorem similar to Schneider's. The proof uses Lemma 5 and the results from the previous chapters.

Theorem 3. Let $L$ be a nilpotent Lie algebra such that $\operatorname{dim}\left(L^{\prime} / L^{\prime \prime}\right)=3$ and $L^{\prime \prime} \neq 0$. Then $L$ can be written as $L=H+U$ where

(i) $H$ is an ideal of $L$ generated by at most five generators; 
(ii) $H^{i}=L^{i}$ for all $i \geq 2$;

(iii) $U$ is an ideal of $L$ such that $U^{\prime} \subseteq L^{5}$;

(iv) $H$ and $U$ centralize each other.

Proof. First, choose the generating set $\left\{a, b, u_{i}, \ldots, u_{r}\right\}$ for $L$ as in Lemma 5. We will modify the set so that in addition to the properties from Lemma 5, one of the following hold:

(a) $u_{1}, u_{2}, \ldots, u_{r} \in C_{L}(a)$ or

(b) $u_{2}, u_{3}, \ldots, u_{r} \in C_{L}\left(\left\langle a, u_{1}\right\rangle\right)$.

If we have condition $(a)$ then we have conditions from Lemma 5 and we are done. So suppose there exists at least one $u_{i} \notin C_{L}(a)$, call it $u_{1}$. So $\left[u_{1}, a\right] \neq 0$ but $\left[u_{1}, a\right] \in L^{5}$ from the Lemma 5 , hence $\left[u_{1}, a\right]=\alpha[a, b, a, a, b]$. Scaling $u_{1}$, we have $\left[u_{1}, a\right]=[a, b, a, a, b]$. Now for $j=2,3, \ldots, r,\left[u_{j}, a\right]=\alpha_{j}[a, b, a, a, b]$, then let $u_{j}^{\prime}=u_{j}-\alpha_{j} u_{1}$. Then

$$
\left[u_{j}^{\prime}, a\right]=\left[u_{j}, a\right]-\alpha_{j}\left[u_{1}, a\right]=\alpha_{j}[a, b, a, a, b]-\alpha_{j}[a, b, a, a, b]=0 .
$$

So we have $u_{2}^{\prime}, u_{3}^{\prime}, \ldots u_{r}^{\prime} \in C_{L}(a)$ and $\left[u_{1}, a\right]=[a, b, a, a, b]$. If all the $u_{j}$ 's centralize $u_{1}$ we are done because we have satistifed the conditions for case (b), so suppose not and say $u_{2}^{\prime}$ does not centralize $u_{1}$. Now $\left[u_{2}^{\prime}, u_{1}\right]=[a, b, a, a, b]$ (scaling $u_{2}$ ) and for $j=3,4, \ldots, r$ we have $\left[u_{j}^{\prime}, u_{1}\right]=\beta_{j}[a, b, a, a, b]$. So let $u_{j}^{\prime \prime}=u_{j}^{\prime}-\beta_{j} u_{2}^{\prime}$. Then

$$
\left[u_{j}^{\prime \prime}, u_{1}\right]=\left[u_{j}^{\prime}, u_{1}\right]-\beta_{j}\left[u_{2}^{\prime}, u_{1}\right]=\beta_{j}[a, b, a, a, b]-\beta_{j}[a, b, a, a, b]=0 .
$$


Now dropping all primes for simplicity we have

$$
\begin{array}{r}
{\left[u_{1}, a\right]=[a, b, a, a, b],} \\
u_{2}, u_{3}, \ldots, u_{5} \in C_{L}(a), \\
{\left[u_{2}, u_{1}\right]=[a, b, a, a, b], \text { and }} \\
u_{3}, u_{4}, \ldots, u_{r} \in C_{L}\left(u_{1}\right) .
\end{array}
$$

If $u_{3}, u_{4}, \ldots, u_{5}$ centralize $u_{2}$ then we go to the next paragraph (at ${ }^{*}$ ). If not, use $u_{3}$ and similarly suppose $\left[u_{3}, u_{2}\right]=[a, b, a, a, b]$ (scaling $u_{3}$ ). Then for $j=4,5, \ldots, r$, $\left[u_{j}, u_{2}\right]=\beta_{j}[a, b, a, a, b]$. So let $u_{j}^{\prime \prime \prime}=u_{j}-\beta_{j} u_{3}$. Then $\left[u_{j}^{\prime \prime \prime}, u_{2}\right]=0$ for $j=4,5, \ldots, r$. Dropping the primes we have

1. $\left[u_{1}, a\right]=[a, b, a, a, b]$

2. $u_{2}, u_{3}, \ldots, u_{r} \in C_{L}(a)$

3. $\left[u_{2}, u_{1}\right]=[a, b, a, a, b]$ and $\left[u_{3}, u_{2}\right]=[a, b, a, a, b]$

4. $u_{3}, u_{4}, \ldots, u_{r} \in C_{L}\left(u_{1}\right)$ and $u_{4}, u_{5}, \ldots, u_{r} \in C_{L}\left(u_{2}\right)$

Continuing in the same process, one more step would produce

1. $\left[u_{1}, a\right]=[a, b, a, a, b]$

2. $u_{2}, u_{3}, \ldots, u_{r} \in C_{L}(a)$

3. $\left[u_{2}, u_{1}\right]=[a, b, a, a, b],\left[u_{3}, u_{2}\right]=[a, b, a, a, b]$ and $\left[u_{4}, u_{3}\right]=[a, b, a, a, b]$

4. $u_{3}, u_{4}, \ldots, u_{r} \in C_{L}\left(u_{1}\right), u_{4}, u_{5}, \ldots, u_{r} \in C_{L}\left(u_{2}\right)$ and $u_{5}, u_{6}, \ldots u_{r} \in C_{L}\left(u_{3}\right)$

So we continue this process until we get to some $k$ such that

1. $\left[u_{1}, a\right]=[a, b, a, a, b]$ 
2. $u_{2}, u_{3}, \ldots, u_{r} \in C_{L}(a)$

3. $\left[u_{i}, u_{i-1}\right]=[a, b, a, a, b]$ for $i=2,3, \ldots, k$

4. $\left[u_{k+1}, u_{k}\right]=0$

5. $u_{i+2}, u_{i+3}, \ldots, u_{r} \in C_{L}\left(u_{i}\right)$ for all $i=1,2, \ldots, k$

If $k$ is even, then let $a^{\prime}=a+u_{2}+u_{4}+\ldots+u_{k}$. Then

$$
\begin{aligned}
{\left[u_{1}, a^{\prime}\right] } & =\left[u_{1}, a\right]+\left[u_{1}, u_{2}\right]+\left[u_{1}, u_{4}\right]+\ldots+\left[u_{1}, u_{k}\right] \\
& =[a, b, a, a, b]-[a, b, a, a, b]+0+\ldots+0 \\
& =0 \\
{\left[u_{2}, a^{\prime}\right] } & =\left[u_{2}, a\right]+\left[u_{2}, u_{2}\right]+\left[u_{2}, u_{4}\right]+\ldots+\left[u_{2}, u_{k}\right] \\
& =0+0++0+\ldots+0 \\
& =0, \text { and } \\
{\left[u_{3}, a^{\prime}\right] } & =\left[u_{3}, a\right]+\left[u_{3}, u_{2}\right]+\left[u_{3}, u_{4}\right]+\ldots+\left[u_{3}, u_{k}\right] \\
& =0+[a, b, a, a, b]-[a, b, a, a, b]+0+\ldots+0 \\
& =0 .
\end{aligned}
$$

Continuing on we see that $u_{1}, u_{2}, \ldots, u_{r} \in C_{L}\left(a^{\prime}\right)$, hence property (a) holds. Now if $k$ is odd, let $u_{1}^{\prime}=u_{1}+u_{3}+\ldots+u_{k}$. Then similarly, we can see that $u_{2}, u_{3}, \ldots, u_{r} \in$ $C_{L}\left(\left\langle a, u_{1}\right\rangle\right)$, hence property (b) holds.

*Now suppose that $\left\{a, b, u_{1}, \ldots, u_{r}\right\}$ is the generating set with the conditions from the previous lemma, and in addition, property (a) holds. First we will assume that all the $u_{i}^{\prime} s$ centralize $b \bmod L^{5}$. If $\left[u_{i}, b\right]=\delta_{i}[a, b, a, a, b]$ for some $i \in\{1,2, \ldots, r\}$, then let $u_{i}^{\prime}=u_{i}-\delta_{i}[a, b, a, a]$. Then $H=\langle a, b\rangle$, and $U=\left\langle u_{1}, u_{2}, \ldots, u_{r}\right\rangle$ (where we have dropped 
the primes) satisfy the assertions of the theorem. That is, $[H, U]=0$, and $L=H+U$. The other conditions follow immediately.

Now suppose that some of the $u_{i}^{\prime} s$ do not centralize $b \bmod L^{5}$, and assume without loss of generality that it is $u_{1}$. Then $\left[u_{1}, b\right]=[a, b, a, a]+\delta_{1}[a, b, a, a, b]$ where we have scaled $u_{1}$ to obtain the a coefficient of 1 for $[a, b, a, a]$. Then let $u_{1}^{\prime}=u_{1}-\delta_{1}[a, b, a, a]$, which implies that

$$
\begin{aligned}
{\left[u_{1}^{\prime}, b\right] } & =\left[u_{1}, b\right]-\delta_{1}[a, b, a, a, b] \\
& =[a, b, a, a]+\delta_{1}[a, b, a, a, b]-\delta_{1}[a, b, a, a, b] \\
& =[a, b, a, a] .
\end{aligned}
$$

Now for $j=2,3, \ldots, r$, the same substitution yields $\left[u_{j}^{\prime}, b\right]=\alpha_{j}[a, b, a, a]$. So let $u_{j}^{\prime \prime}=$ $u_{j}^{\prime}-\alpha_{j} u_{1}^{\prime}$ for $j=2,3, \ldots r$. Then

$$
\left[u_{j}^{\prime \prime}, b\right]=\left[u_{j}^{\prime}, b\right]-\alpha_{j}\left[u_{1}^{\prime}, b\right]=\alpha_{j}[a, b, a, a]-\alpha_{j}[a, b, a, a]=0
$$

Dropping the primes for simplicity we have

$$
\begin{aligned}
& {\left[u_{1}, b\right]=[a, b, a, a] \text { and }} \\
& u_{2}, u_{3}, \ldots, u_{r} \in C_{L}(b) .
\end{aligned}
$$

If $u_{2}, u_{3}, \ldots, u_{r}$ centralize $u_{1}$, then choose $H=\left\langle a, b, u_{1}\right\rangle$ and $U=\left\langle u_{2}, u_{3}, \ldots, u_{r}\right\rangle$ and we are done. So, suppose this is not the case and $\left[u_{2}, u_{1}\right]=[a, b, a, a, b]$. Then we can repeat the first part of the proof and select a generating set $\left\{a, b, u_{1}, u_{2}, \ldots, u_{r}\right\}$ such that the following properties hold:

1. $\left[u_{1}, b\right]=[a, b, a, a]$ 
2. $u_{2}, u_{3}, \ldots, u_{r} \in C_{L}(b)$

3. $\left[u_{k-1}, u_{k-2}\right]=[a, b, a, a, b]$

4. $\left[u_{k+1}, u_{k}\right]=0$

5. $u_{i+2}, u_{i+3}, \ldots, u_{r} \in C_{L}\left(u_{i}\right)$ for all $i=1,2, \ldots, k$

If $k$ is even, then we can set $H=\left\langle a, b, u_{1}+u_{3}+\ldots+u_{k-1}, u_{2}+u_{4}+\ldots+u_{k}\right\rangle$ and $U=\left\langle u_{2}, u_{3}, \ldots, u_{k-1}, u_{k+1}, \ldots, u_{r}\right\rangle$. If $k$ is odd, set $H=\left\langle a, b, u_{1}+u_{3}+\ldots+u_{k}\right\rangle$ and $U=\left\langle u_{2}, u_{3}, \ldots, u_{r}\right\rangle$. In both cases, $L=H+U$ and $[H, U]=0$, so $H$ and $U$ satisfy the assertions of the theorem.

In the case of property (b), we consider the Lie algebra $L_{1}=\left\langle a, b, u_{2}, \ldots, u_{r}\right\rangle$ and choose subalgebras $H_{1}$ and $U_{1}$ according to the process described previously. Then again, $L_{1}=H_{1}+U_{1}$ and $\left[H_{1}, U_{1}\right]=0$, and so $H_{1}$ and $U_{1}$ satisfy the assertions of the theorem. Moreover, $H_{1}$ can be generated by at most four elements. For $L$, we can choose the subalgebras $H=\left\langle H_{1}, u_{1}\right\rangle$ and $U=U_{1}$.

Lemma 5 and Theorem 3 give the Lie algebra analog to the Schneider's central decomposition. Here we have found that $L=H+U$, where we will see in the next chapters the structure of $U$ and that $H$ is one of fourteen algebras after we classify $H$ over the complex numbers. 


\section{Chapter 5}

\section{The Structure of $\mathrm{U}$}

The nilpotent Lie algebra $\mathrm{U}$ in Theorem 3 has the multiplication of a skew form from $U$ into $\langle z\rangle$, where $z=[a, b, a, a, b]$. $U$ has a basis $x_{1}, y_{1}, x_{2}, y_{2}, \ldots, x_{r}, y_{r}, z, w_{1}, \ldots, w_{s}$ where $\left[x_{i}, y_{j}\right]=\delta_{i j} z$ and $Z(U)=\left\langle z, w_{1}, \ldots, w_{s}\right\rangle$ by Theorem 6.3 [3]. Hence $U$ is the direct sum of a generalized Heisenberg Lie algebra made up by the $x, y$, and $z^{\prime} s$, and an abelian Lie algebra made up by the $w^{\prime} s$. 


\section{Chapter 6}

\section{Classification of $\mathrm{L}=\mathrm{H}+\mathrm{U}$ when \\ $\mathbf{H}=\langle a, b\rangle$}

We now turn to classifying the Lie algebra $L=H+U$ given in Theorem 3 . For the remainder of this paper, we will examine the possible structures of the Lie algebra $H$ over the complex numbers. We consider the cases as the number of generators for $H$ increase. $H$ is said to be degenerate if $H=H_{1}+U_{1}$ where $H_{1}$ and $U_{1}$ are as in Theorem 3 and $U_{1} \neq 0$, and do not consider them further. To begin, we will consider $H=\langle a, b\rangle$.

When $H$ is generated by two elements, we know $U$ is empty, $H$ has dimension 6 , and $L^{6}=0$ since $\operatorname{dim}\left(L^{\prime \prime}\right)=1$. If we say $H$ is generated by $a$ and $b$ as in the previous chapters, then we have $[[a, b],[a, b]]=[a, b, a, b]+[a,[a, b, b]]$. Since the left hand side is 0 and $[a,[b,[a, b]]]=0$ since $[a, b, b] \in H^{4}$ from Lemma 3 , it follows that $[a, b, a, b]=0$. Also, $[[a, b, a],[a, b]]=[a, b, a, a, b]+[a,[a, b, a, b]]=[a, b, a, a, b]$. Note that since $[a, b, b] \in H^{4}$, $[a, b, b]=e[a, b, a, a]+f[a, b, a, a, b]$. Thus we can construct the following multiplication table: 
Table 6.1: Two Generated Multiplication Table

\begin{tabular}{|c|c|c|c|c|c|c|}
\hline & $\mathbf{a}$ & $\mathbf{b}$ & {$[\mathbf{a}, \mathbf{b}]$} & {$[\mathbf{a}, \mathbf{b}, \mathbf{a}]$} & {$[\mathbf{a}, \mathbf{b}, \mathbf{a}, \mathbf{a}]$} & {$[\mathbf{a}, \mathbf{b}, \mathbf{a}, \mathbf{a}, \mathbf{b}]$} \\
\hline $\mathbf{a}$ & 0 & {$[\mathrm{a}, \mathrm{b}]$} & $-[\mathrm{a}, \mathrm{b}, \mathrm{a}]$ & $-[\mathrm{a}, \mathrm{b}, \mathrm{a}, \mathrm{a}]$ & 0 & 0 \\
\hline $\mathbf{b}$ & $-[\mathrm{a}, \mathrm{b}]$ & 0 & $* *$ & 0 & $-[\mathrm{a}, \mathrm{b}, \mathrm{a}, \mathrm{a}, \mathrm{b}]$ & 0 \\
\hline$[\mathbf{a}, \mathbf{b}]$ & {$[\mathrm{a}, \mathrm{b}, \mathrm{a}]$} & $* *$ & 0 & $-[\mathrm{a}, \mathrm{b}, \mathrm{a}, \mathrm{a}, \mathrm{b}]$ & 0 & 0 \\
\hline$[\mathbf{a}, \mathbf{b}, \mathbf{a}]$ & {$[\mathrm{a}, \mathrm{b}, \mathrm{a}, \mathrm{a}]$} & 0 & {$[\mathrm{a}, \mathrm{b}, \mathrm{a}, \mathrm{a}, \mathrm{b}]$} & 0 & 0 & 0 \\
\hline$[\mathbf{a}, \mathbf{b}, \mathbf{a}, \mathbf{a}]$ & 0 & {$[\mathrm{a}, \mathrm{b}, \mathrm{a}, \mathrm{a}, \mathrm{b}]$} & 0 & 0 & 0 & 0 \\
\hline$[\mathbf{a}, \mathbf{b}, \mathbf{a}, \mathbf{a}, \mathbf{b}]$ & 0 & 0 & 0 & 0 & 0 & 0 \\
\hline
\end{tabular}

Where ${ }^{* *}$ is given by the fact that since $[a, b, b] \in H^{4},[a, b, b]=e[a, b, a, a]+$ $f[a, b, a, a, b]$. If a second algebra of this type with generators $\alpha, \beta$ has the identity $[\alpha, \beta, \beta]=s[\alpha, \beta, \alpha, \alpha]+t[\alpha, \beta, \alpha, \alpha, \beta]$, we would like to know when these algebras are isomorphic. We look for a change of basis by setting

$$
\begin{aligned}
& \alpha=c_{0} a+c_{1} b+c_{2}[a, b]+c_{3}[a, b, a]+c_{4}[a, b, a, a]+c_{5}[a, b, a, a, b] \\
& \beta=h_{1} b+h_{2}[a, b]+h_{3}[a, b, a]+h_{4}[a, b, a, a]+h_{5}[a, b, a, a, b]
\end{aligned}
$$

where $c_{0}, h_{1} \neq 0$. Multiplying these together we obtain the following expressions:

- $[\alpha, \beta]=c_{0} h_{1}[a, b]-c_{0} h_{2}[a, b, a]-c_{0} h_{3}[a, b, a, a]-c_{1} h_{2}[a, b, b]-c_{1} h_{4}[a, b, a, a, b]+$ $c_{2} h_{1}[a, b, b]-c_{2} h_{3}[a, b, a, a, b]+c_{3} h_{2}[a, b, a, a, b]+c_{4} h_{1}[a, b, a, a, b]$

- $[\alpha, \beta, \alpha]=c_{0}^{2} h_{1}[a, b, a]-c_{0}^{2} h_{2}[a, b, a, a]+c_{0} c_{1} h_{1}[a, b, b]-c_{0} c_{1} h_{3}[a, b, a, a, b]$ - $c_{1}^{2} h_{2}[a, b, b, b]-c_{0} c_{2} h_{2}[a, b, a, a, b]-c_{0} c_{3} h_{1}[a, b, a, a, b]+c_{1} c_{2} h_{1}[a, b, b, b]$

- $[\alpha, \beta, \alpha, \alpha]=c_{0}^{3} h_{1}[a, b, a, a]-c_{0}^{2} c_{1} h_{2}[a, b, a, a, b]+c_{0} c_{1}^{2} h_{1}[a, b, b, b]$ $+c_{0}^{2} c_{2} h_{1}[a, b, a, a, b]$

- $[\alpha, \beta, \alpha, \alpha, \alpha]=c_{0}^{3} c_{1} h_{1}[a, b, a, a, b]$

- $[\alpha, \beta, \alpha, \alpha, \beta]=c_{0}^{3} h_{1}^{2}[a, b, a, a, b]$

- $[[\alpha, \beta, \alpha], \beta]=c_{0} c_{1} h_{1}^{2}[a, b, b, b]$ 
- $[[\alpha, \beta],[\alpha, \beta, \alpha]]=-c_{0}^{3} h_{1}^{2}[a, b, a, a, b]$

- $[\alpha, \beta, \beta]=c_{0} h_{1}^{2}[a, b, b]-c_{0} h_{1} h_{3}[a, b, a, a, b]-c_{1} h_{1} h_{2}[a, b, b, b]+c_{2} h_{1}^{2}[a, b, b, b]$

- $c_{0} h_{2}^{2}[a, b, a, a, b]-c_{0} h_{1} h_{3}[a, b, a, a, b]$

- $[\alpha, \beta, \beta, \beta]=c_{0} h_{1}^{3}[a, b, b, b]$

Note that since we know $c_{0}, h_{1} \neq 0$, and we have $0=[\alpha, \beta, \alpha, \alpha, \alpha]=c_{0}^{3} c_{1} h_{1}[a, b, a, a, b]$, therefore $c_{1}=0$. Now since $[\alpha, \beta, \beta]=s[\alpha, \beta, \alpha, \alpha]+t[\alpha, \beta, \alpha, \alpha, \beta]$ it follows that $[\alpha, \beta, \beta, \beta]=s[\alpha, \beta, \alpha, \alpha, \beta]$, and so $c_{0} h_{1}^{3}[a, b, b, b]=s c_{0}^{3} h_{1}^{2}[a, b, a, a, b]$ or $c_{0} h_{1}^{3} e[a, b, a, a, b]=s c_{0}^{3} h_{1}^{2}[a, b, a, a, b]$. Hence

$$
e c_{0} h_{1}^{3}=s c_{0}^{3} h_{1}^{2} \text { oreh } h_{1}=s c_{0}^{2}
$$

Hence $e=0$ if and only if $s=0$. Now suppose $e=s=0$, and then let $t=0$. Then in the algebra generated by $\alpha$ and $\beta$ we get $[\alpha, \beta, \beta]=0$, and in the algebra generated by $a$ and $b$ we get $[a, b, b]=f[a, b, a, a, b]$. Now set $c_{0}=h_{1}=1$ and $h_{3}=\frac{f}{2}$ and all other $c_{i}$ and $h_{j}=0$. Then $\alpha=a, \beta=b+\frac{f}{2}[a, b, a]$ and

$$
\begin{aligned}
{[\alpha, \beta] } & =[a, b]-\frac{f}{2}[a, b, a, a] \\
{[\alpha, \beta, \beta] } & =[a, b, b]-\frac{f}{2}[a, b, a, a, b]+\frac{f}{2}[[a, b],[a, b, a]] \\
& =[a, b, b]-f[a, b, a, a, b]=0
\end{aligned}
$$

and any algebra with $[a, b, b] \in H^{5}$ is isomorphic to one such that $[\alpha, \beta, \beta]=0$ and we obtain the following multiplication table: 
Table 6.2: Two Generated Multiplication Table Case 1

\begin{tabular}{|c|c|c|c|c|c|c|}
\hline & $\mathbf{a}$ & $\mathbf{b}$ & {$[\mathbf{a}, \mathbf{b}]$} & {$[\mathbf{a}, \mathbf{b}, \mathbf{a}]$} & {$[\mathbf{a}, \mathbf{b}, \mathbf{a}, \mathbf{a}]$} & {$[\mathbf{a}, \mathbf{b}, \mathbf{a}, \mathbf{a}, \mathbf{b}]$} \\
\hline $\mathbf{a}$ & 0 & {$[\mathrm{a}, \mathrm{b}]$} & $-[\mathrm{a}, \mathrm{b}, \mathrm{a}]$ & $-[\mathrm{a}, \mathrm{b}, \mathrm{a}, \mathrm{a}]$ & 0 & 0 \\
\hline $\mathbf{b}$ & $-[\mathrm{a}, \mathrm{b}]$ & 0 & 0 & 0 & $-[\mathrm{a}, \mathrm{b}, \mathrm{a}, \mathrm{a}, \mathrm{b}]$ & 0 \\
\hline$[\mathbf{a}, \mathbf{b}]$ & {$[\mathrm{a}, \mathrm{b}, \mathrm{a}]$} & 0 & 0 & $-[\mathrm{a}, \mathrm{b}, \mathrm{a}, \mathrm{a}, \mathrm{b}]$ & 0 & 0 \\
\hline$[\mathbf{a}, \mathbf{b}, \mathbf{a}]$ & {$[\mathrm{a}, \mathrm{b}, \mathrm{a}, \mathrm{a}]$} & 0 & {$[\mathrm{a}, \mathrm{b}, \mathrm{a}, \mathrm{a}, \mathrm{b}]$} & 0 & 0 & 0 \\
\hline$[\mathbf{a}, \mathbf{b}, \mathbf{a}, \mathbf{a}]$ & 0 & {$[\mathrm{a}, \mathrm{b}, \mathrm{a}, \mathrm{a}, \mathrm{b}]$} & 0 & 0 & 0 & 0 \\
\hline$[\mathbf{a}, \mathbf{b}, \mathbf{a}, \mathbf{a}, \mathbf{b}]$ & 0 & 0 & 0 & 0 & 0 & 0 \\
\hline
\end{tabular}

Now suppose that $e, s \neq 0$ and set $s=1, t=0$ so that $[\alpha, \beta, \beta]=[\alpha, \beta, \alpha, \alpha]$. We can show any algebra with $e \neq 0$ is isomorphic to one with this product by setting $c_{0}=$ $1, h_{1}=\frac{1}{e}, h_{3}=\frac{f}{2 e}$, and all other $c_{i}, h_{j}=0$. By doing this, then $\alpha=a, \beta=\frac{1}{e} b+\frac{f}{2 e}[a, b, a]$, and we get $[\alpha, \beta]=\frac{1}{e}[a, b]-\frac{f}{2 e}[a, b, a, a]$ so $[\alpha, \beta, \beta]=\frac{1}{e^{2}}[a, b, b]-\frac{f}{2 e^{2}}[a, b, a, a, b]-$ $\frac{f}{2 e^{2}}[a, b, a, a, b]=[\alpha, \beta, \alpha, \alpha]$. Hence this change of basis gives the identity for any of these algebras with $s \neq 0$, and we get the multiplication table:

Table 6.3: Two Generated Multiplication Table Case 2

\begin{tabular}{|c|c|c|c|c|c|c|}
\hline & $\mathbf{a}$ & $\mathbf{b}$ & {$[\mathbf{a}, \mathbf{b}]$} & {$[\mathbf{a}, \mathbf{b}, \mathbf{a}]$} & {$[\mathbf{a}, \mathbf{b}, \mathbf{a}, \mathbf{a}]$} & {$[\mathbf{a}, \mathbf{b}, \mathbf{a}, \mathbf{a}, \mathbf{b}]$} \\
\hline $\mathbf{a}$ & 0 & {$[\mathrm{a}, \mathrm{b}]$} & $-[\mathrm{a}, \mathrm{b}, \mathrm{a}]$ & $-[\mathrm{a}, \mathrm{b}, \mathrm{a}, \mathrm{a}]$ & 0 & 0 \\
\hline $\mathbf{b}$ & $-[\mathrm{a}, \mathrm{b}]$ & 0 & $-[\mathrm{a}, \mathrm{b}, \mathrm{a}, \mathrm{a}]$ & 0 & $-[\mathrm{a}, \mathrm{b}, \mathrm{a}, \mathrm{a}, \mathrm{b}]$ & 0 \\
\hline$[\mathbf{a}, \mathbf{b}]$ & {$[\mathrm{a}, \mathrm{b}, \mathrm{a}]$} & {$[\mathrm{a}, \mathrm{b}, \mathrm{a}, \mathrm{a}]$} & 0 & $-[\mathrm{a}, \mathrm{b}, \mathrm{a}, \mathrm{a}, \mathrm{b}]$ & 0 & 0 \\
\hline$[\mathbf{a}, \mathbf{b}, \mathbf{a}]$ & {$[\mathrm{a}, \mathrm{b}, \mathrm{a}, \mathrm{a}]$} & 0 & {$[\mathrm{a}, \mathrm{b}, \mathrm{a}, \mathrm{a}, \mathrm{b}]$} & 0 & 0 & 0 \\
\hline$[\mathbf{a}, \mathbf{b}, \mathbf{a}, \mathbf{a}]$ & 0 & {$[\mathrm{a}, \mathrm{b}, \mathrm{a}, \mathrm{a}, \mathrm{b}]$} & 0 & 0 & 0 & 0 \\
\hline$[\mathbf{a}, \mathbf{b}, \mathbf{a}, \mathbf{a}, \mathbf{b}]$ & 0 & 0 & 0 & 0 & 0 & 0 \\
\hline
\end{tabular}

Summarizing, if $H$ is a two-generated Lie algebra with $\operatorname{dim}\left(H^{\prime} / H^{\prime \prime}\right)=3, H^{\prime \prime} \neq$ 0 with the same notation as before, the basis for $H$ is given by $\{a, b,[a, b],[a, b, a]$, $[a, b, a, a],[a, b, a, a, b]\}$, and $\operatorname{dim}(H)=6 . \quad$ Also, $H^{2}=H^{\prime}=\langle[a, b],[a, b, a],[a, b, a, a]$, $[a, b, a, a, b]\rangle, \operatorname{dim}\left(H^{\prime}\right)=4, H^{3}=\langle[a, b, a],[a, b, a, a],[a, b, a, a, b]\rangle, H^{4}=\langle[a, b, a, a]$, $[a, b, a, a, b]\rangle, H^{5}=H^{\prime \prime}=\{[a, b, a, a, b]\}, \operatorname{dim}\left(H^{\prime \prime}\right)=1, \mathrm{Z}(H)=\{[a, b, a, a, b]\}$, and $H^{6}=0$ so $H$ is nilpotent of class 5 . We summarize these results into the following theorem.

Theorem 4. Suppose $H$ is a two generated nilpotent Lie algebra with $\operatorname{dim}\left(H^{\prime} / H^{\prime \prime}\right)=3$ and $H^{\prime \prime} \neq 0$. Then there exists generators $a$ and $b$ such that the multiplication table 
between basis elements is given either in Table 6.2 or Table 6.3. These algebras are not isomorphic since in one case $H^{\prime} / H^{5}$ is abelian and in the other case it is not. 


\section{Chapter 7}

\section{Isomorphism Classes when \\ $\mathbf{H}=\langle a, b, u\rangle$}

Now consider $H=\langle a, b, u\rangle$ where $M=\langle a, b\rangle$ is the 2-generated algebra as in the previous chapter, and let $y=[a, b, a, a]$ and $z=[a, b, a, a, b]$ for simplicity. From Lemma $5,[u, a]=\alpha z,[u, b]=\beta y+\gamma z$ and $u \notin C_{H}(M)$, where $\alpha, \beta, \gamma \in \mathbb{C}$ and $\mathbb{C}$ is the complex numbers. We will try to divide these algebras into isomorphism classes, each with a simple representation by changing $u$. Let $u^{\prime}=\rho y+\tau u$, with $\tau \neq 0$, otherwise $u^{\prime} \in M$. Then

$$
\begin{gathered}
{\left[u^{\prime}, a\right]=\tau[u, a]=\tau \alpha z \text { and }} \\
{\left[u^{\prime}, b\right]=\rho z+\tau(\beta y+\gamma z)}
\end{gathered}
$$

Let $\rho=-\tau \gamma$. Then $\left[u^{\prime}, b\right]=\tau \beta y$ and $\left[u^{\prime}, a\right]=\tau \alpha z$. We now alter $\tau$ by looking at all the possible cases for $\alpha$ and $\beta$, which are:

1. If $\alpha=0$ and $\beta=0$, then $u^{\prime} \in C_{H}(M)$ and $H=M+U$, therefore $H$ is degenerate.

2. If $\alpha=0$ and $\beta \neq 0$, then let $\tau=\frac{1}{\beta}$. Then $\left[u^{\prime}, a\right]=0$ and $\left[u^{\prime}, b\right]=y$. 
3. If $\alpha \neq 0$ and $\beta=0$, then let $\tau=\frac{1}{\alpha}$. Then $\left[u^{\prime}, a\right]=z$ and $\left[u^{\prime}, b\right]=0$.

4. If $\alpha \neq 0$ and $\beta \neq 0$, then let $\tau=\frac{1}{\alpha}$. Then $\left[u^{\prime}, a\right]=z$ and $\left[u^{\prime}, b\right]=\sigma y$ where $\sigma=\frac{\beta}{\alpha}$.

In the last case, we want $\sigma=1$ so we rescale $a, b$ and $u^{\prime}$ to produce a further simplification. By letting $a^{\prime}=d a, b^{\prime}=e b$ and $u^{\prime \prime}=f u^{\prime}$ where $d, e$, and $f$ are scalars then $\left[a^{\prime}, b^{\prime}\right]=d e[a, b],\left[a^{\prime}, b^{\prime}, a^{\prime}\right]=d^{2} e[a, b, a],\left[a^{\prime}, b^{\prime}, a^{\prime}, a^{\prime}\right]=y^{\prime}=d^{3} e y,\left[a^{\prime}, b^{\prime}, a^{\prime}, a^{\prime}, b^{\prime}\right]=$ $z^{\prime}=d^{3} e^{2} z$, and $\left[a^{\prime}, b^{\prime}, b^{\prime}\right]=d e^{2}[a, b, b]$. Also, $\left[u^{\prime \prime}, a^{\prime}\right]=d f\left[u^{\prime}, a\right]=d f z=\frac{d f}{d^{3} e^{2}} z^{\prime}$ and $\left[u^{\prime \prime}, b^{\prime}\right]=\frac{f e \sigma}{d^{3} e} y^{\prime}$.

Either $[a, b, b]=0$ or $[a, b, b]=y$. In the first case $\left[a^{\prime}, b^{\prime}, b^{\prime}\right]=d e^{2}[a, b, b]=0$ and we are done. In the second case $\left[a^{\prime}, b^{\prime}, b^{\prime}\right]=d e^{2} y=\frac{d e^{2}}{d^{3} e} y^{\prime}=\frac{e}{d^{2}} y^{\prime}$. Now we choose $e, d$ and $f$ such that $\frac{f}{d^{2} e^{2}}=1, \frac{f}{d^{3}} \sigma=1$, and $\frac{e}{d^{2}}=1$. We accomplish this by letting $d=\frac{1}{\sigma^{1 / 3}}, f=\frac{1}{\sigma^{2}}$ and $e=\frac{1}{\sigma^{2 / 3}}$.

Dropping all primes we have the following 6 possible isomorphism classes:

Table 7.1: Possible Isomorphisms

\begin{tabular}{|l|l|}
\hline 1 & {$[\mathrm{u}, \mathrm{a}]=\mathrm{z},[\mathrm{u}, \mathrm{b}]=0,[\mathrm{a}, \mathrm{b}, \mathrm{b}]=0$} \\
\hline 2 & {$[\mathrm{u}, \mathrm{a}]=0,[\mathrm{u}, \mathrm{b}]=\mathrm{y},[\mathrm{a}, \mathrm{b}, \mathrm{b}]=0$} \\
\hline 3 & {$[\mathrm{u}, \mathrm{a}]=\mathrm{z},[\mathrm{u}, \mathrm{b}]=\mathrm{y},[\mathrm{a}, \mathrm{b}, \mathrm{b}]=0$} \\
\hline 4 & {$[\mathrm{u}, \mathrm{a}]=\mathrm{z},[\mathrm{u}, \mathrm{b}]=0,[\mathrm{a}, \mathrm{b}, \mathrm{b}]=\mathrm{y}$} \\
\hline 5 & {$[\mathrm{u}, \mathrm{a}]=0,[\mathrm{u}, \mathrm{b}]=\mathrm{y},[\mathrm{a}, \mathrm{b}, \mathrm{b}]=\mathrm{y}$} \\
\hline 6 & {$[\mathrm{u}, \mathrm{a}]=\mathrm{z},[\mathrm{u}, \mathrm{b}]=\mathrm{y},[\mathrm{a}, \mathrm{b}, \mathrm{b}]=\mathrm{y}$} \\
\hline
\end{tabular}

In this case, $H$ is degenerate precisely when $\operatorname{dim}(Z(H))>1$. Directly checking the six cases we find that they are all non degenerate. We would now like to examine which of these cases are isomorphic to each other. Notice that $H=\{a, b,[a, b],[a, b, a], y, z, u\}$, $H^{2}=\{[a, b],[a, b, a], y, z\}, H^{1}=\{[a, b],[a, b, a], y, z, b, u\}$, and $Z(H)=H^{5}=\{z\}$. In cases 1,2 , and $3, C_{H}\left(H^{2} / H^{5}\right)=\{b,[a, b],[a, b, a], y, z, u\}$ and in cases 4,5 and 6 $C_{H}\left(H^{2} / H^{5}\right)=\{[a, b],[a, b, a], y, z, u\}$. Since the dimensions are not equal, we can conclude that cases 1,2 , and 3 are not isomorphic to cases 4,5 , and 6 . Now by examining $C_{H}\left(H^{1} / H^{5}\right)$ we get that in case $1, C_{H}\left(H^{1} / H^{5}\right)=\{b,[a, b],[a, b, a], y, z, u\}$, in cases 2 
and $3 C_{H}\left(H^{1} / H^{5}\right)=\{[a, b],[a, b, a], y, z\}$, in case $4, C_{H}\left(H^{1} / H^{5}\right)=\{[a, b, a], y, z, u\}$ and in cases 5 and $6, C_{H}\left(H^{1} / H^{5}\right)=\{[a, b, a], y, z\}$. Again, by counting the dimensions we can conclude that case 1 is not isomorphic to case 2 or 3 , and case 4 is not isomorphic to case 5 or 6 . It remains to show the relationship between cases 2 and 3, and between cases 5 and 6.

Suppose that case 2 is represented with primes and case 3 is not and that they are isomorphic. Let $a^{\prime}=c_{0} a+c_{1} b+c_{2}[a, b]+c_{3}[a, b, a]+c_{4} y+c_{5} z+c_{6} u$ and $b^{\prime}=$ $h_{1} b+h_{2}[a, b]+h_{3}[a, b, a]+h_{4} y+h_{5} z+h_{6} u$ similar to equations (6.1) and (6.2). Let $u^{\prime}=d_{0} a+d_{1} b+d_{2}[a, b]+d_{3}[a, b, a]+d_{4} y+d_{5} z+d_{6} u$, and note that $\left[u^{\prime}, H^{2}\right]=0$ since $\left[u^{\prime},\left[a^{\prime}, b^{\prime}\right]\right]=\left[\left[u^{\prime}, a^{\prime}\right], b^{\prime}\right]+\left[a^{\prime},\left[u^{\prime}, b^{\prime}\right]\right]=0$. Now $0=\left[u^{\prime},[a, b]\right]=d_{0}[a,[a, b]]+d_{1}[b,[a, b]]+$ $d_{3}[a, b, a, a, b]$ which implies that $d_{0}=d_{3}=0$. Also, $0=\left[u^{\prime},[a, b, a]\right]=-d_{2}[a, b, a, a, b]$ so $d_{2}=0$ and $0=\left[u^{\prime},[a, b, a, a]\right]=-d_{1} z$, hence $d_{1}=0$. Therefore $u^{\prime}=d_{4} y+d_{5} z+d_{6} u$. Now

$$
0=\left[u^{\prime}, a^{\prime}\right]=d_{4} c_{1} z+d_{6} c_{0}[u, a]+d_{6} c_{1}[u, b]
$$

Thus $0=d_{4} c_{1}+d_{6} c_{0}$. But $c_{1}=0$ from previous chapter 6 work and $c_{0} \neq 0$ hence $d_{6}=0$ and $u^{\prime} \in\langle a, b\rangle$ which is a contradiction. By similar reasoning we can conclude cases 5 and 6 are also not isomorphic. Therefore, we have six distinct isomorphism classes when $H=\langle a, b, u\rangle$. Summarizing this case into the following theorem:

Theorem 5. When $H$ is a nilpotent Lie algebra such that $\operatorname{dim}\left(H^{\prime} / H^{\prime \prime}\right)=3, H^{\prime \prime} \neq 0$ and $H=\langle a, b, u\rangle$ with $a$ and $b$ as in the previous theorem and $u \notin C_{H}(\langle a, b\rangle)$, then there are 6 isomorphism classes whose canonical algebras are given in Table \%.1. 


\section{Chapter 8}

\section{Isomorphism Classes for \\ $H=\left\langle a, b, u_{1}, u_{2}\right\rangle$}

Now consider the case $H=\left\langle a, b, u_{1}, u_{2}\right\rangle$, where $H$ is as in Theorem 3 and $M=$ $\langle a, b\rangle$ as in Table 6.2 or Table 6.3. Again let $y=[a, b, a, a]$ and $z=[a, b, a, a, b]$ for simplification. From Lemma 5, $\left[u_{1}, a\right]=\alpha_{1} z,\left[u_{1}, b\right]=\beta_{1} y+\gamma_{1} z,\left[u_{2}, a\right]=\alpha_{2} z,\left[u_{2}, b\right]=$ $\beta_{2} y+\gamma_{2} z$. Next we will divide the algebras into isomorphism classes, each with a simple multiplication between basis elements. Let

$$
\begin{aligned}
& u_{1}^{\prime}=c_{0} y+c_{1} u_{1}+c_{2} u_{2} \\
& u_{2}^{\prime}=d_{0} y+d_{1} u_{1}+d_{2} u_{2}
\end{aligned}
$$

where $\left[\begin{array}{cc}c_{1} & c_{2} \\ d_{1} & d_{2}\end{array}\right]$ is non-singular. Then 


$$
\begin{aligned}
& {\left[u_{1}^{\prime}, a\right]=\left(c_{1} \alpha_{1}+c_{2} \alpha_{2}\right) z=\rho_{1} z} \\
& {\left[u_{2}^{\prime}, a\right]=\left(d_{1} \alpha_{1}+d_{2} \alpha_{2}\right) z=\rho_{2} z} \\
& {\left[u_{1}^{\prime}, b\right]=c_{0}[y, b]+c_{1}\left(\beta_{1} y+\gamma_{1} z\right)+c_{2}\left(\beta_{2} y+\gamma_{2} z\right)=\sigma_{1} y+\tau_{1} z} \\
& {\left[u_{2}^{\prime}, b\right]=d_{0}[y, b]+d_{1}\left(\beta_{1} y+\gamma_{1} z\right)+d_{2}\left(\beta_{2} y+\gamma_{2} z\right)=\sigma_{2} y+\tau_{2} z}
\end{aligned}
$$

Setting $\tau_{1}=\tau_{2}=0$ and then letting $c_{0}=-c_{1} \gamma_{1}-c_{2} \gamma_{2}$ and $d_{0}=-d_{1} \gamma_{1}-d_{2} \gamma_{2}$ we obtain that both $\left[u_{1}^{\prime}, b\right],\left[u_{2}^{\prime}, b\right] \in\langle y\rangle$.

Placing our equations into a matrix, we have $\left[\begin{array}{cc}c_{1} & c_{2} \\ d_{1} & d_{2}\end{array}\right]\left[\begin{array}{cc}\alpha_{1} & \beta_{1} \\ \alpha_{2} & \beta_{2}\end{array}\right]=\left[\begin{array}{cc}\rho_{1} & \sigma_{1} \\ \rho_{2} & \sigma_{2}\end{array}\right]$ which we denote by $A B=C$.

If $\operatorname{rank}(\mathrm{B})=2$, then we can solve $A B=I$ to obtain

$$
\left[u_{1}^{\prime}, a\right]=z,\left[u_{1}^{\prime}, b\right]=0,\left[u_{2}^{\prime}, a\right]=0, \text { and }\left[u_{2}^{\prime}, b\right]=y
$$

It is impossible for $\operatorname{rank}(\mathrm{B})=0$, otherwise $u_{1}, u_{2} \in U$ as in Theorem 3 .

If $\operatorname{rank}(B)=1$, we have 3 cases:

1. If the second column of $\mathrm{B}$ is 0 , we solve $A B=\left[\begin{array}{ll}1 & 0 \\ 0 & 0\end{array}\right]$ to obtain

$$
\left[u_{1}^{\prime}, a\right]=z,\left[u_{1}^{\prime}, b\right]=0,\left[u_{2}^{\prime}, a\right]=0, \text { and }\left[u_{2}^{\prime}, b\right]=0
$$

2. If the first column of $B$ is 0 , we solve $A B=\left[\begin{array}{ll}0 & 1 \\ 0 & 0\end{array}\right]$ to obtain

$$
\left[u_{1}^{\prime}, a\right]=0,\left[u_{1}^{\prime}, b\right]=y,\left[u_{2}^{\prime}, a\right]=0, \text { and }\left[u_{2}^{\prime}, b\right]=0
$$


3. If neither column of $\mathrm{B}$ is 0 , then we can solve $A B=\left[\begin{array}{ll}1 & \delta \\ 0 & 0\end{array}\right]$ for some $\delta \neq 0$ to obtain

$$
\left[u_{1}^{\prime}, a\right]=z,\left[u_{1}^{\prime}, b\right]=\delta y,\left[u_{2}^{\prime}, a\right]=0 \text {, and }\left[u_{2}^{\prime}, b\right]=0
$$

Another change will allow us to take $\delta=1$ for further simplification. Dropping all primes in the foregoing to simplify the notation, let $a^{\prime}=d a, b^{\prime}=e b$, and $u_{1}^{\prime}=f u_{1}$ where no scalar is 0 . Then we compute

$$
\begin{gathered}
{\left[a^{\prime}, b^{\prime}\right]=d e[a, b]} \\
{\left[a^{\prime}, b^{\prime}, a^{\prime}\right]=d^{2} e[a, b, a]} \\
y^{\prime}=\left[a^{\prime}, b^{\prime}, a^{\prime}, a^{\prime}\right]=d^{3} e y \\
z^{\prime}=d^{3} e^{2} z \\
{\left[a^{\prime}, b^{\prime}, b^{\prime}\right]=d e^{2}[a, b, b]} \\
{\left[u_{1}^{\prime}, a^{\prime}\right]=d f\left[u_{1}, a\right]=d f z=\frac{d f}{d^{3} e^{2}} z^{\prime}=\frac{f}{d^{2} e^{2}} z^{\prime}} \\
{\left[u_{1}^{\prime}, b^{\prime}\right]=e f\left[u_{1}, b\right]=e f \delta y=\frac{e f \delta}{d^{3} e} y^{\prime}=\frac{f \delta}{d^{3}} y^{\prime}}
\end{gathered}
$$

Furthermore, $[a, b, b]=0$ or $y$. Hence, $\left[a^{\prime}, b^{\prime}, b^{\prime}\right]=0$ or $\left[a^{\prime}, b^{\prime}, b^{\prime}\right]=d e^{2} y=\frac{e}{d^{2}} y^{\prime}$. Therefore we need to solve

$$
\frac{f}{d^{2} e^{2}}=1, \frac{f \delta}{d^{3}}=1 \text { and } \frac{e}{d^{2}}=1
$$

Set $d=\frac{1}{\delta^{1 / 3}}, e=\frac{1}{\delta^{2 / 3}}$ and $f=\frac{1}{\delta^{2}}$ to satisfy the above equations. Then, by dropping all primes the third $\operatorname{rank}(\mathrm{B})=1$ case becomes

$$
\left[u_{1}, a\right]=z,\left[u_{1}, b\right]=y,\left[u_{2}, a\right]=0,\left[u_{2}, b\right]=0 \text { and }[a, b, b]=0 \text { or } y
$$


Since $u_{1}$ and $u_{2}$ have been altered, now $\left[u_{1}, u_{2}\right]=\pi z$. A final change will allow $\pi=0$ or $\pi=1$ to be used. In all $\operatorname{rank}(\mathrm{B})=1$ cases $\pi \neq 0$ and we can let $u_{2}^{\prime}=\frac{1}{\pi} u_{2}$. Since $\left[u_{2}, a\right]=\left[u_{2}, b\right]=0$, we can add $\left[u_{1}, u_{2}\right]=z$ to the multiplication list. In the $\operatorname{rank}(\mathrm{B})=2$ case, let $a^{\prime}=d a, b^{\prime}=e b, u_{1}^{\prime}=f_{1} u_{1}, u_{2}^{\prime}=f_{2} u_{2}$. As before, $y^{\prime}=d^{3} e y$ and $z^{\prime}=d^{3} e^{2} z$,

$$
\begin{gathered}
{\left[u_{1}^{\prime}, a^{\prime}\right]=d f_{1}\left[u_{1}, a\right]=d f_{1} z=\frac{d f_{1}}{d^{3} e^{2}} z^{\prime}} \\
{\left[u_{2}^{\prime}, b^{\prime}\right]=e f_{2}\left[u_{2}, b\right]=e f_{2} y=\frac{e f_{2}}{d^{3} e} y} \\
{\left[u_{1}^{\prime}, u_{2}^{\prime}\right]=f_{1} f_{2}\left[u_{1}, u_{2}\right]=f_{1} f_{2} \pi z=\frac{f_{1} f_{2}}{d^{3} e^{2}} \pi z^{\prime}} \\
{\left[a^{\prime}, b^{\prime}, b^{\prime}\right]=\frac{d e^{2}}{d^{3} e} y^{\prime}=\frac{e}{d^{2}} y^{\prime}}
\end{gathered}
$$

We want

$$
\frac{e}{d^{2}}=1, \frac{f_{1} f_{2}}{d^{3} e^{2}} \pi=1, \frac{f_{1}}{d^{2} e^{2}}=1 \text { and } \frac{f_{2}}{d^{3}}=1
$$

Let $d=\frac{1}{\pi^{1 / 2}}, e=\frac{1}{\pi}, f_{1}=\frac{1}{\pi^{3}}$ and $f_{2}=\frac{1}{\pi^{3 / 2}}$ to solve these equations. Then

$$
\begin{gathered}
{\left[u_{1}^{\prime}, a^{\prime}\right]=z^{\prime},\left[u_{1}^{\prime}, b^{\prime}\right]=y^{\prime},\left[u_{2}^{\prime}, a^{\prime}\right]=0,\left[u_{2}^{\prime}, b^{\prime}\right]=0} \\
{\left[u_{1}^{\prime}, u_{2}^{\prime}\right]=z^{\prime} \text { or } 0 \text { and }\left[a^{\prime}, b^{\prime}, b^{\prime}\right]=y^{\prime} \text { or } 0}
\end{gathered}
$$

In summary we have the following 10 possible isomorphism classes (at the top of the next page): 
Table 8.1: Five Generated Possible Isomorphisms

\begin{tabular}{|c|l|}
\hline 1 & {$\left[u_{1}, a\right]=z,\left[u_{1}, b\right]=0,\left[u_{2}, a\right]=0,\left[u_{2}, b\right]=y,\left[u_{1}, u_{2}\right]=z,[a, b, b]=0$} \\
\hline 2 & {$\left[u_{1}, a\right]=z,\left[u_{1}, b\right]=0,\left[u_{2}, a\right]=0,\left[u_{2}, b\right]=y,\left[u_{1}, u_{2}\right]=0,[a, b, b]=0$} \\
\hline 3 & {$\left[u_{1}, a\right]=z,\left[u_{1}, b\right]=0,\left[u_{2}, a\right]=0,\left[u_{2}, b\right]=0,\left[u_{1}, u_{2}\right]=z,[a, b, b]=0$} \\
\hline 4 & {$\left[u_{1}, a\right]=0,\left[u_{1}, b\right]=y,\left[u_{2}, a\right]=0,\left[u_{2}, b\right]=0,\left[u_{1}, u_{2}\right]=z,[a, b, b]=0$} \\
\hline 5 & {$\left[u_{1}, a\right]=z,\left[u_{1}, b\right]=y,\left[u_{2}, a\right]=0,\left[u_{2}, b\right]=0,\left[u_{1}, u_{2}\right]=z,[a, b, b]=0$} \\
\hline 6 & {$\left[u_{1}, a\right]=z,\left[u_{1}, b\right]=0,\left[u_{2}, a\right]=0,\left[u_{2}, b\right]=y,\left[u_{1}, u_{2}\right]=z,[a, b, b]=y$} \\
\hline 7 & {$\left[u_{1}, a\right]=z,\left[u_{1}, b\right]=0,\left[u_{2}, a\right]=0,\left[u_{2}, b\right]=y,\left[u_{1}, u_{2}\right]=0,[a, b, b]=y$} \\
\hline 8 & {$\left[u_{1}, a\right]=z,\left[u_{1}, b\right]=0,\left[u_{2}, a\right]=0,\left[u_{2}, b\right]=0,\left[u_{1}, u_{2}\right]=z,[a, b, b]=y$} \\
\hline 9 & {$\left[u_{1}, a\right]=0,\left[u_{1}, b\right]=y,\left[u_{2}, a\right]=0,\left[u_{2}, b\right]=0,\left[u_{1}, u_{2}\right]=z,[a, b, b]=y$} \\
\hline 10 & {$\left[u_{1}, a\right]=z,\left[u_{1}, b\right]=y,\left[u_{2}, a\right]=0,\left[u_{2}, b\right]=0,\left[u_{1}, u_{2}\right]=z,[a, b, b]=y$} \\
\hline
\end{tabular}

We will now divide these 10 classes into isomorphism classes. Similar to when $H=$ $\langle a, b, u\rangle$, we see that cases 1-5 are when $[a, b, b]=0$ and cases 6-10 are when $[a, b, b]=y$ and bases for $H, H^{1}, H^{\prime}=H^{2}$ and $H^{5}$ are

$$
\begin{aligned}
H & =\left\{a, b,[a, b],[a, b, a], y, z, u_{1}, u_{2}\right\} \\
H^{1} & =\left\{b,[a, b],[a, b, a], y, z, u_{1}, u_{2}\right\} \\
H^{2} & =\{[a, b],[a, b, a], y, z\} \\
H^{5} & =\{z\}
\end{aligned}
$$

Notice for cases 3 and 8 , if we let $\hat{a}=a-u_{2}$, then $\left[u_{1}, \hat{a}\right]=\left[u_{1}, a\right]-\left[u_{1}, u_{2}\right]=z-z=0$, so then $u_{1}, u_{2} \in U$ and these cases really belong in the two generator case, so they are degenerate. We now check to see if any of the remaining 8 cases are degenerate in the sense that $H=H_{1}+U_{1}, U_{1} \neq 0$ and $H_{1}, U_{1}$ are as in Theorem 3 .

If $\operatorname{dim}(Z(H))=2$ or 3 , then $H$ is clearly degenerate. We will find $\operatorname{dim}(Z(H))$ by letting $x=c_{0} a+c_{1} b+c_{2}[a, b]+c_{3}[a, b, a]+c_{4} y+c_{5} z+c_{6} u_{1}+c_{7} u_{2} \in Z(H)$. First consider cases $1,2,6$ and 7 where $\left[u_{2}, b\right]=y$. Then $[x, a]=c_{1}[a, b]+c_{2}[a, b, a]+c_{3} y+c_{6} z=0$ and $[x, b]=c_{0}[a, b]+c_{4} z+c_{7} y=0$. Hence $c_{0}=c_{1}=c_{2}=c_{3}=c_{4}=c_{6}=c_{7}=0$ and $\operatorname{dim}(Z(H))=1$. Now consider $Z\left(H / H^{5}\right)$. Let $x+H^{5} \in Z\left(H / H^{5}\right)$ with $x$ as above. 
Then $[x, a]=c_{1}[b, a]+c_{2}[a, b, a]+c_{3} y \in H^{5}$ and $[x, b]=c_{0}[a, b]+c_{7} y \in H^{5}$. Then $c_{1}=c_{2}=c_{3}=c_{0}=c_{7}=0$ and $x=c_{4} y+c_{6} u_{1}$ and $\operatorname{dim}\left(Z\left(H / H^{5}\right)\right)=2$.

Suppose that $H=H_{1}+U_{1}$. If $\operatorname{dim}\left(U_{1}\right)=1$, then $\operatorname{dim}(Z(H))=2$, which is a contradiction. If $\operatorname{dim}\left(U_{1}\right)=2$, then $\operatorname{dim}\left(Z\left(H / H^{5}\right)\right)=3$, which is also a contradiction. Hence all 4 cases are nondegenerate. Cases 4, 5, 9 and 10 are handled in a similar manner and all 4 of these cases are also nondegenerate.

Now that we have determined which cases are degenerate, we now divide the remaining 8 nondegenerate cases into isomorphism classes. In cases $1-5$, we find $C_{H}\left(H^{2} / H^{5}\right)$ $=\left\{b,[a, b],[a, b, a], y, z, u_{1}, u_{2}\right\}$ so the dimension is 7. However, in cases 6-10 we find $C_{H}\left(H^{2} / H^{5}\right)=\left\{[a, b],[a, b, a], y, z, u_{1}, u_{2}\right\}$ so the dimension is 6 . Therefore cases $1-5$ are not isomorphic to cases 6-10. Also, $C_{H}\left(H^{1}\right)=\left\{z, u_{1}\right\}$ in cases 2 and 7 and $C_{H}\left(H^{1}\right)=\{z\}$ in cases 1, 4, 5, 6, 9 and 10. Thus the class defined by 2 and the one defined by 7 are not isomorphic to any of the other classes. In summary we have cases $1,2,4,5$ are not isomorphic to cases $6,7,9$ and 10, and case 2 is not isomorphic to cases 1 , 4, and 5, and case 7 is not isomorphic to cases 6,9 and 10 . It remains to see if cases 1,4 , and 5 are isomorphic as well as if cases 6, 9, and 10 are isomorphic.

We claim that case 5 is isomorphic to case $4(5 \cong 4)$. Let $a, b, u_{1}, u_{2}$ give the multiplication table for class 5 . If we let $\bar{a}=a-u_{2}$, then $[\bar{a}, b]=\left[a-u_{2}, b\right]=[a, b],[\bar{a}, b, \bar{a}]=$ $\left[a, b, a-u_{2}\right]=[a, b, a], \bar{y}=[\bar{a}, b, \bar{a}, \bar{a}]=\left[a, b, a, a-u_{2}\right]=y,[\bar{a}, b, \bar{a}, \bar{a}, \bar{a}]=[a, b, a, a, a]=$ $0, \bar{z}=[\bar{a}, b, \bar{a}, \bar{a}, b]=[a, b, a, a, b]=z,\left[u_{1} \bar{a}\right]=\left[u_{1}, a-u_{2}\right]=\left[u_{1}, a\right]-\left[u_{1}, u_{2}\right]=z-z=$ $0,\left[u_{1}, b\right]=y,\left[u_{2}, \bar{a}\right]=\left[u_{2}, a-u_{2}\right]=\left[u_{2}, a\right]=0,\left[u_{2}, b\right]=0,\left[u_{1}, u_{2}\right]=z$ and $[\bar{a}, b, b]=$ $[a, b, b]=0$, so we have $5 \cong 4$. Similarly, with the same change we can also show that $10 \cong 9$, since all of the above equations are the same and $[\bar{a}, b, b]=[a, b, b]=y$.

We also claim that $1 \cong 4$. Let $a, b, u_{1}, u_{2}$ give the multiplication for case 4 , and now we will show we can change to get the multiplication in 1 . If we let $\bar{a}=a+u_{1}-u_{2}, \bar{b}=$ 
$b-[a, b]+u_{2}, \overline{u_{1}}=-u_{2}$, and $\overline{u_{2}}=-y+u_{1}-u_{2}$ then

$$
\begin{gathered}
{\left[\overline{u_{1}}, u_{2}\right]=-\left[u_{2}, u_{1}\right]=z} \\
{[\bar{a}, \bar{b}, \bar{b}]=[a, b, b]+\left[u_{1}, b, b\right]+[a,-[a, b],-[a, b]]=0+[y, b]+[a,[a, b],[a, b]]=z-z=0} \\
{\left[\overline{u_{1}}, \bar{a}\right]=\left[-u_{2}, a+u_{1}-u_{2}\right]=z=\bar{z}} \\
{\left[\overline{u_{1}}, \bar{b}\right]=\left[-u_{2}, b-[a, b]+u_{2}\right]=0} \\
{\left[\overline{u_{2}}, \bar{a}\right]=\left[-y+u_{1}-u_{2}, a+u_{1}-u_{2}\right]=-\left[u_{1}, u_{2}\right]-\left[u_{2}, u_{1}\right]=0} \\
{\left[\overline{u_{2}}, \bar{b}\right]=\left[-y+u_{1}-u_{2}, b-[a, b]+u_{2}\right]=-z+y+z=\bar{y}}
\end{gathered}
$$

Thus this change takes type 4 into an algebra with multiplication

$$
\left[u_{1}, a\right]=z,\left[u_{1}, b\right]=0,\left[u_{2}, a\right]=0,\left[u_{2}, b\right]=y,\left[u_{1}, u_{2}\right]=z,[a, b, b]=0
$$

Hence $4 \cong 1$. The same transformation shows $9 \cong 6$. Hence $1 \cong 4 \cong 5$ and $6 \cong 9 \cong 10$.

Therefore we have 4 isomorphism classes when $H=\left\langle a, b, u_{1}, u_{2}\right\rangle$. They have representations as shown in type 1, 2, 6 and 7.

Theorem 6. Let $H=\left\langle a, b, u_{1}, u_{2}\right\rangle$ as in Theorem 3. Then there are four isomorphism classes represented by the multiplication formulas of types 1, 2, 6 and 7 in Table 8.1. 


\section{Chapter 9}

\section{Isomorphism Classes for}

$$
H=\left\langle a, b, u_{1}, u_{2}, u_{3}\right\rangle
$$

Finally we consider $H=\left\langle a, b, u_{1}, u_{2}, u_{3}\right\rangle$, where $H$ is as in Theorem 3 and $M=\langle a, b\rangle$ as in Table 6.2 or Table 6.3. Continue to let $y=[a, b, a, a]$ and $z=[a, b, a, a, b]$, we have $\left[u_{1}, a\right]=\alpha_{1} z,\left[u_{1}, b\right]=\beta_{1} y+\gamma_{1} z,\left[u_{2}, a\right]=\alpha_{2} z,\left[u_{2}, b\right]=\beta_{2} y+\gamma_{2} z,\left[u_{3}, a\right]=\alpha_{3} z$, $\left[u_{3}, b\right]=\beta_{3} y+\gamma_{3} z$. Let

$$
\begin{aligned}
& u_{1}^{\prime}=c_{0} y+c_{1} u_{1}+c_{2} u_{2}+c_{3} u_{3} \\
& u_{2}^{\prime}=d_{0} y+d_{1} u_{1}+d_{2} u_{2}+d_{3} u_{3} \\
& u_{3}^{\prime}=e_{0} y+e_{1} u_{1}+e_{2} u_{2}+e_{3} u_{3}
\end{aligned}
$$

where placing the coefficients in the following non-singular matrix $\left[\begin{array}{ccc}c_{1} & c_{2} & c_{3} \\ d_{1} & d_{2} & d_{3} \\ e_{1} & e_{2} & e_{3}\end{array}\right]$. 
Then

$$
\begin{aligned}
& {\left[u_{1}^{\prime}, a\right]=c_{1} \alpha_{1} z+c_{2} \alpha_{2} z+c_{3} \alpha_{3} z=\rho_{1} z} \\
& {\left[u_{2}^{\prime}, a\right]=d_{1} \alpha_{1} z+d_{2} \alpha_{2} z+d_{3} \alpha_{3} z=\rho_{2} z} \\
& {\left[u_{3}^{\prime}, a\right]=e_{1} \alpha_{1} z+e_{2} \alpha_{2} z+e_{3} \alpha_{3} z=\rho_{3} z} \\
& {\left[u_{1}^{\prime}, b\right]=c_{0} z+c_{1}\left(\beta_{1} y+\gamma_{1} z\right)+c_{2}\left(\beta_{2} y+\gamma_{2} z\right)+c_{3}\left(\beta_{3} y+\gamma_{3} z\right)=\sigma_{1} y+\tau_{1} z} \\
& {\left[u_{2}^{\prime}, b\right]=d_{0} z+d_{1}\left(\beta_{1} y+\gamma_{1} z\right)+d_{2}\left(\beta_{2} y+\gamma_{2} z\right)+d_{3}\left(\beta_{3} y+\gamma_{3} z\right)=\sigma_{2} y+\tau_{2} z} \\
& {\left[u_{3}^{\prime}, b\right]=e_{0} z+e_{1}\left(\beta_{1} y+\gamma_{1} z\right)+e_{2}\left(\beta_{2} y+\gamma_{2} z\right)+e_{3}\left(\beta_{3} y+\gamma_{3} z\right)=\sigma_{3} y+\tau_{3} z}
\end{aligned}
$$

Set $\tau_{1}=\tau_{2}=\tau_{3}=0$. Then letting $c_{0}=-c_{1} \gamma_{1}-c_{2} \gamma_{2}-c_{3} \gamma_{3}, d_{0}=-d_{1} \gamma_{1}-d_{2} \gamma_{2}-d_{3} \gamma_{3}$, and $e_{0}=-e_{1} \gamma_{1}-e_{2} \gamma_{2}-e_{3} \gamma_{3}$ we obtain that $\left[u_{1}^{\prime}, b\right],\left[u_{2}^{\prime}, b\right],\left[u_{3}^{\prime}, b\right] \in\langle y\rangle$. Simplifying the equations into a matrix equation to solve, we have

$$
\left[\begin{array}{lll}
c_{1} & c_{2} & c_{3} \\
d_{1} & d_{2} & d_{3} \\
e_{1} & e_{2} & e_{3}
\end{array}\right]\left[\begin{array}{ll}
\alpha_{1} & \beta_{1} \\
\alpha_{2} & \beta_{2} \\
\alpha_{3} & \beta_{3}
\end{array}\right]=\left[\begin{array}{ll}
\rho_{1} & \sigma_{1} \\
\rho_{2} & \sigma_{2} \\
\rho_{3} & \sigma_{3}
\end{array}\right]
$$

which we denote by $A B=C$ where $A$ is non-singular. The analysis follows the same pattern as in the last chapter.

$$
\begin{aligned}
& \text { If } \operatorname{rank}(\mathrm{B})=2 \text {, then we can find A such that } A B=\left[\begin{array}{cc}
1 & 0 \\
0 & 1 \\
0 & 0
\end{array}\right] \text { to obtain } \\
& \qquad\left[u_{1}^{\prime}, a\right]=z,\left[u_{1}^{\prime}, b\right]=0,\left[u_{2}^{\prime}, a\right]=0,\left[u_{2}^{\prime}, b\right]=y,\left[u_{3}^{\prime}, a\right]=0 \text { and }\left[u_{3}^{\prime}, b\right]=0
\end{aligned}
$$

It is impossible for $\operatorname{rank}(\mathrm{B})=0$ for then $u_{1}, u_{2}, u_{3} \in U$ as in Theorem 3 . If $\operatorname{rank}(B)=1$, similar to the last chapter, we obtain 3 cases: 
1. $\left[u_{1}, a\right]=z,\left[u_{1}, b\right]=0,\left[u_{2}, a\right]=0,\left[u_{2}, b\right]=0,\left[u_{3}, a\right]=0$, and $\left[u_{3}, b\right]=0$

2. $\left[u_{1}, a\right]=0,\left[u_{1}, b\right]=y,\left[u_{2}, a\right]=0,\left[u_{2}, b\right]=0,\left[u_{3}, a\right]=0$, and $\left[u_{3}, b\right]=0$

3. $\left[u_{1}, a\right]=z,\left[u_{1}, b\right]=\delta y,\left[u_{2}, a\right]=0,\left[u_{2}, b\right]=0,\left[u_{3}, a\right]=0$ and $\left[u_{3}, b\right]=0$ where $\delta \neq 0$

In case 3 , just like before, we change generators to make $\delta=1$. Let $a^{\prime}=d a, b^{\prime}=e b$, and $u_{1}^{\prime}=f u_{1}$. The same computations as in the last chapter show that letting $d=\delta^{1 / 3}$, $e=\frac{1}{\delta^{2 / 3}}$, and $f=\frac{1}{\delta^{2}}$ changes case 3 to $\left[u_{1}^{\prime}, a^{\prime}\right]=z$ and $\left[u_{1}^{\prime}, b^{\prime}\right]=y$ and the other brackets are 0 . To proceed, we drop the primes for simplicity and now case 3 becomes

$$
\left[u_{1}, a\right]=z,\left[u_{1}, b\right]=y,\left[u_{2}, a\right]=0,\left[u_{2}, b\right]=0,\left[u_{3}, a\right]=0 \text {, and }\left[u_{3}, b\right]=0
$$

Now for each of the above cases we have $\left[u_{1}, u_{2}\right]=\pi_{1} z,\left[u_{1}, u_{3}\right]=\pi_{2} z$ and $\left[u_{2}, u_{3}\right]=$ $\pi_{3} z$. For the rank 1 case, if $\pi_{1}=0$ we can interchange $u_{2}$ and $u_{3}$, then $\pi_{1} \neq 0$. Now let $\hat{u_{2}}=\frac{1}{\pi_{1}} u_{2}$, then $\left[u_{1}, \hat{u_{2}}\right]=\frac{1}{\pi_{1}}\left[u_{1}, u_{2}\right]=z$. Dropping the hat we have $\left[u_{1}, u_{2}\right]=z,\left[u_{1}, u_{3}\right]=$ $\pi_{2} z,\left[u_{2}, u_{3}\right]=\pi_{3} z$. Now we can let $\hat{u_{3}}=u_{3}-\pi_{2} u_{2}$ and then $\left[u_{1}, \hat{u_{3}}\right]=\pi_{2} z-\pi_{2} z=0$ and $\left[u_{2}, \hat{u_{3}}\right]=\left[u_{2}, u_{3}\right]-\pi_{2}\left[u_{2}, u_{2}\right]=\left[u_{2}, u_{3}\right]$. So dropping the hat again, we now have $\left[u_{1}, u_{2}\right]=z,\left[u_{1}, u_{3}\right]=0,\left[u_{2}, u_{3}\right]=\pi_{3} z$. Now $\pi_{3} \neq 0$, otherwise we drop down into a lower dimensional case, and hence this case is degenerate. But if we let $\hat{u_{3}}=\frac{1}{\pi_{3}} u_{3}$ and change back to the $u^{\prime} s$ we have $\left[u_{1}, u_{2}\right]=z,\left[u_{1}, u_{3}\right]=0,\left[u_{2}, u_{3}\right]=z$. Then we can let $\hat{u_{1}}=u_{3}+u_{1}$ and then $\left[\hat{u}_{1}, u_{3}\right]=\left[u_{3}, u_{3}\right]+\left[u_{1}, u_{3}\right]=0$ and $\left[\hat{u_{1}}, u_{2}\right]=\left[u_{3}, u_{2}\right]+\left[u_{1}, u_{2}\right]=-z+z=0$ so then both $u_{2}$ and $u_{3}$ drop out and we are again in a lower dimensional case. Hence all rank 1 cases are degenerate.

For the $\operatorname{rank}(B)=2$ case, we have

$$
\left[u_{1}, a\right]=z,\left[u_{1}, b\right]=0,\left[u_{2}, a\right]=0,\left[u_{2}, b\right]=y,\left[u_{3}, a\right]=0,\left[u_{3}, b\right]=0
$$




$$
\left[u_{1}, u_{2}\right]=\pi_{1} z,\left[u_{1}, u_{3}\right]=\pi_{2} z, \text { and }\left[u_{2}, u_{3}\right]=\pi_{3} z
$$

Note that $\pi_{2}$ and $\pi_{3}$ cannot both be 0 , otherwise $u_{3} \in U$ and we would be back in the 8 dimensional case.

Suppose that $\pi_{3}=0$. Then $\pi_{2} \neq 0$. Let $a^{\prime}=d a, b^{\prime}=e b, u_{1}^{\prime}=f_{1} u_{1}, u_{2}^{\prime}=f_{2} u_{2}$ and $u_{3}^{\prime}=f_{3} u_{3}$. Then $\left[a^{\prime}, b^{\prime}\right]=d e[a, b],\left[a^{\prime}, b^{\prime}, a^{\prime}\right]=d^{2} e[a, b, a], y^{\prime}=\left[a^{\prime}, b^{\prime}, a^{\prime}, a^{\prime}\right]=d^{3} e y, z^{\prime}=$ $\left[a^{\prime}, b^{\prime}, a^{\prime}, a^{\prime}, b^{\prime}\right]=d^{3} e^{2} z$

$$
\begin{gathered}
{\left[a^{\prime}, b^{\prime}, b^{\prime}\right]=d e^{2}[a, b, b]=d e^{2} y=\frac{e}{d^{2}} y^{\prime} \text { or } 0} \\
{\left[u_{1}^{\prime}, a^{\prime}\right]=f_{1} d z=\frac{f_{1} d}{d^{3} e^{2}} z^{\prime}} \\
{\left[u_{2}^{\prime}, b^{\prime}\right]=f_{2} e y=\frac{f_{2} e}{d^{3} e} y^{\prime}} \\
{\left[u_{1}^{\prime}, u_{2}^{\prime}\right]=f_{1} f_{2}\left[u_{1}, u_{2}\right]=\frac{f_{1} f_{2}}{d^{3} e^{2}} \pi_{1} z^{\prime}} \\
{\left[u_{1}^{\prime}, u_{3}^{\prime}\right]=\frac{f_{1} f_{3}}{d^{3} e^{2}} \pi_{2} z^{\prime} \pi_{2} \neq 0}
\end{gathered}
$$

If $\pi_{1} \neq 0$ we want $\frac{e}{d^{2}}=\frac{f_{1}}{d^{2} e^{2}}=\frac{f_{2}}{d^{3}}=\frac{f_{1} f_{2} \pi_{1}}{d^{3} e^{2}}=\frac{f_{1} f_{3} \pi_{2}}{d^{3} e^{2}}=1$. Let $d=\frac{1}{\sqrt{\pi_{1}}}, e=\frac{1}{\pi_{1}}, f_{1}=\frac{1}{\pi_{1}^{3}}$, $f_{2}=\frac{1}{\pi_{1}^{3 / 2}}$, and $f_{3}=\frac{1}{\left(\pi_{1}\right)^{1 / 2} \pi_{2}}$ and these equations are satisfied. Thus, dropping the primes, $\left[u_{1}, u_{2}\right]=z,\left[u_{1}, u_{3}\right]=z$ and $\left[u_{2}, u_{3}\right]=0$. Now let $u_{2}^{\prime}=u_{2}-u_{3}$. We get that $\left[u_{1}, u_{2}^{\prime}\right]=0,\left[u_{2}, u_{2}^{\prime}\right]=0,\left[u_{3}, u_{2}^{\prime}\right]=0$ and $\left[u_{2}, b\right]=y$. Then if we let $a^{\prime}=a-u_{3}$, we also get $\left[u_{1}, a^{\prime}\right]=0,\left[u_{2}^{\prime}, a^{\prime}\right]=0$ and $\left[u_{3}, a^{\prime}\right]=0$. Hence, dropping the primes $u_{1}, u_{3} \in U$ and this case is degenerate.

If $\pi_{1}=0,\left(\right.$ and therefore $\pi_{3}=0$ ), then we want $\frac{e}{d^{2}}=\frac{f_{1}}{d^{2} e^{2}}=\frac{f_{2}}{d^{3}}=\frac{f_{1} f_{3} \pi_{2}}{d^{3} e^{2}}=1$. So let $f_{3}=\frac{1}{\pi_{2}}, f_{1}=f_{2}=d=e=1$ to satisfy these equations, and when we drop the primes we obtain $\left[u_{1}, u_{2}\right]=0,\left[u_{1}, u_{3}\right]=z$ and $\left[u_{2}, u_{3}\right]=0$. Set $a^{\prime}=a-u_{3}$ and we have $\left[u_{1}, a^{\prime}\right]=0$ which implies $u_{1}, u_{3} \in U$. Hence this case is also degenerate.

Thus far, all cases have been degenerate. There remains the $\operatorname{rank}(\mathrm{B})=2$ case with 
$\pi_{3} \neq 0$. Notice that if $\pi_{1} \neq 0$, we can let $u_{1}=\pi_{3} u_{1}+\pi_{1} u_{3}$. Then $\left[u_{1}^{\prime}, u_{2}\right]=\left[\pi_{3} u_{1}+\right.$ $\left.\pi_{1} u_{3}, u_{2}\right]=\left(\pi_{3} \pi_{1}-\pi_{1} \pi_{3}\right) z=0$. Therefore we can assume that $\pi_{1}=0$. Notice that this change forces $\left[u_{1}, a\right]=\rho z, \rho \neq 0$. Therefore we have

$$
\begin{gathered}
{\left[u_{1}, a\right]=\rho z,\left[u_{1}, b\right]=0,\left[u_{2}, a\right]=0,\left[u_{2}, b\right]=y,\left[u_{3}, a\right]=0,\left[u_{3}, b\right]=0} \\
{\left[u_{1}, u_{2}\right]=0,\left[u_{1}, u_{3}\right]=\pi_{2} z, \text { and }\left[u_{2}, u_{3}\right]=\pi_{3} z}
\end{gathered}
$$

Once again let $a^{\prime}=d a, b^{\prime}=e b, u_{1}^{\prime}=f_{1} u_{1}, u_{2}^{\prime}=f_{2} u_{2}$, and $u_{3}^{\prime}=f_{3} u_{3}$. As before we have $y^{\prime}=d^{3} e y, z^{\prime}=d^{3} e^{2} z,\left[a^{\prime}, b^{\prime}, b^{\prime}\right]=\frac{d e^{2}}{d^{3} e} y^{\prime}$ or $=0,\left[u_{1}^{\prime}, a^{\prime}\right]=\frac{\rho f_{1} d}{d^{3} e^{2}} z^{\prime},\left[u_{2}^{\prime}, b^{\prime}\right]=\frac{f_{2} e}{d^{3} e} y^{\prime}$, $\left[u_{1}^{\prime}, u_{3}^{\prime}\right]=\frac{f_{1} f_{3}}{d^{3} e^{2}} \pi_{2} z^{\prime}$, and $\left[u_{2}^{\prime}, u_{3}^{\prime}\right]=\frac{f_{2} f_{3}}{d^{3} e^{2}} \pi_{3} z^{\prime}$. Again we want to solve for the coefficients. First, let $\pi_{2} \neq 0$. Then we want

$$
\frac{\rho f_{1}}{d^{2} e^{2}}=\frac{f_{2}}{d^{3}}=\frac{f_{1} f_{3} \pi_{2}}{d^{3} e^{2}}=\frac{f_{2} f_{3} \pi_{3}}{d^{3} e^{2}}=\frac{e}{d^{2}}=1
$$

Letting $d=\left(\frac{\rho \pi_{3}}{\pi_{2}}\right)^{1 / 3}, e=\left(\frac{\rho \pi_{3}}{\pi_{2}}\right)^{2 / 3}, f_{2}=\frac{\rho \pi_{3}}{\pi_{2}}, f_{1}=\frac{\rho \pi_{3}^{2}}{\pi_{2}^{2}}$, and $f_{3}=\frac{1}{\pi_{3}}\left(\frac{\rho \pi_{3}}{\pi_{2}}\right)^{4 / 3}$ solves the above equations. Hence we get $\left[u_{1}, a\right]=z,\left[u_{1}, b\right]=0,\left[u_{2}, a\right]=0,\left[u_{2}, b\right]=y,\left[u_{3}, a\right]=$ $0,\left[u_{3}, b\right]=0\left[u_{1}, u_{2}\right]=0,\left[u_{1}, u_{3}\right]=z,\left[u_{2}, u_{3}\right]=z$, and $[a, b, b]=0$ or $y$. Let $a^{\prime}=a-u_{3}$ and $u_{2}^{\prime}=u_{2}-u_{1}$. Then $u_{1}, u_{3} \in U$ and this case becomes degenerate.

Finally, let $\pi_{2}=0$. Then we seek a solution to

$$
\frac{\rho f_{1}}{d^{2} e^{2}}=\frac{f_{2}}{d^{3}}=\frac{f_{2} f_{3} \pi_{3}}{d^{3} e^{2}}=\frac{e}{d^{2}}=1
$$

A solution is $d=e=f_{2}=1, f_{1}=\frac{1}{\rho}$ and $f_{3}=\frac{1}{\pi_{3}}$. Note that $\rho$ and $\pi_{3}$ are not 0 . We need to show that this case is nondegenerate. We begin by stating that we know $\operatorname{dim}(Z(H))=$ 1 and suppose that $H$ is degenerate. Let $v_{1}=c_{1} a+c_{2} b+c_{3}[a, b]+c_{4}[a, b, a]+c_{5} y+c_{6} z+$ $c_{7} u_{1}+c_{8} u_{2}+c_{9} u_{3}$ and $v_{2}=d_{1} a+d_{2} b+d_{3}[a, b]+d_{4}[a, b, a]+d_{5} y+d_{6} z+d_{7} u_{1}+d_{8} u_{2}+d_{9} u_{3}$. 
If $v_{1}$ and $v_{2} \in U$, then $\left[v_{1}, M^{2}\right]=0$ and $\left[v_{2}, M^{2}\right]=0$. Now $\left[v_{1},[a, b]\right]=c_{1}[a,[a, b]]+$ $c_{2}[a, b, b]+c_{4} z$ which implies that $c_{1}=c_{4}=0,\left[v_{1},[a, b, a]\right]=c_{3} z$. This implies $c_{3}=0$ and $\left[v_{1}, y\right]=c_{2}[b, y]=0$, so $c_{2}=0$. Similarly, $d_{1}=d_{2}=d_{3}=d_{4}=0$. Now by scaling back the coefficients, we let $a^{\prime}=a+e_{2} b+e_{3}[a, b]+e_{4}[a, b, a]+e_{5} y+e_{6} z+e_{7} u_{1}+e_{8} u_{2}+e_{9} u_{3}$ and $b^{\prime}=b+f_{3}[a, b]+f_{4}[a, b, a]+f_{5} y+f_{6} z+f_{7} u_{1}+f_{8} u_{2}+f_{9} u_{3}$ then

$$
\begin{gathered}
0=\left[v_{1}, b^{\prime}\right]=-c_{8}\left[u_{2}, b\right]+c_{5} z+\left(c_{9} f_{8}-c_{8} f_{9}\right) z, \text { so } c_{8}=0 \text { and similarly } \\
d_{8}=0 \text { and therefore } \\
{\left[v_{1}, v_{2}\right]=\left(c_{8} d_{9}-d_{8} c_{9}\right)\left[u_{2}, u_{3}\right]=0 \text { since } c_{8}=d_{8}=0}
\end{gathered}
$$

This implies that $v_{1}, v_{2} \in Z(H)$ which is a contradiction. So this case is nondegenerate. Hence the multiplication is

Table 9.1: Isomorphism Classes

\begin{tabular}{|c|c|}
\hline$\left[u_{1}, a\right]=z$ & {$\left[u_{1}, b\right]=0$} \\
\hline$\left[u_{2}, a\right]=0$ & {$\left[u_{2}, b\right]=y$} \\
\hline$\left[u_{3}, a\right]=0$ & {$\left[u_{3}, b\right]=0$} \\
\hline$\left[u_{1}, u_{2}\right]=0$ & {$\left[u_{1}, u_{3}\right]=0$} \\
\hline$\left[u_{2}, u_{3}\right]=z$ & {$[a, b, b]=0$ or $y$} \\
\hline
\end{tabular}

Therefore we are left with only two isomorphism classes in the case when $\operatorname{dim}(H)=9$. We summarize this case into the following theorem:

Theorem 7. Let $H=\left\langle a, b, u_{1}, u_{2}, u_{3}\right\rangle$ as in Theorem 3. Then there are two isomorphism classes represented by the multiplication formulas in Table 9.1.

If we combine all of our results, we can formalize one more theorem. 
Theorem 8. Let $L$ be a nilpotent Lie algebra such that $\operatorname{dim}\left(L^{\prime} / L^{\prime \prime}\right)=3$ and $L^{\prime \prime} \neq 0$. Then $L$ can be written as $L=H+U$ as in Theorem 3, where $U$ is the direct sum of a generalized Heisenberg Lie algebra and an abelian Lie algebra, and $H$ can be classified over the complex numbers into one of fourteen isomorphism classes. A further breakdown reveals that there are 2 isomorphism classes when $\operatorname{dim}(H)=6,6$ isomorphism classes when $\operatorname{dim}(H)=7,4$ isomorphism classes when $\operatorname{dim}(H)=8$, and 2 isomorphism classes when $\operatorname{dim}(H)=9$. 


\section{Chapter 10}

\section{Examples}

Here will will provide some examples of the 6 dimensional and 7 dimensional cases of L.

Example 1. Let $L$ be the subalgebra in $T(6, F)$ generated by $A=E_{12}+E_{34}+E_{45}$ and $B=E_{23}+E_{56}$. Then to generate the algebra, need to bracket all elements together until we have everything. So

- $[A, B]=\left(E_{12}+E_{34}+E_{45}\right)\left(E_{23}+E_{56}\right)-\left(E_{23}+E_{56}\right)\left(E_{12}+E_{34}+E_{45}\right)=E_{13}+E_{46}-E_{24}$

- $[A, B, A]=2 E_{14}-E_{25}-E_{36}$

- $[A, B, A, A]=3 E_{15}$

- $[A, B, A, A, A]=0$

- $[A, B, B]=0$

- $[A, B, A, B]=0$

- $[A, B, A, A, B]=3 E_{16}$

- $[[A, B, A],[A, B]]=3 E_{16}$ 
Here the dimension of $L$ is 6 , the basis for $L=\{A, B,[A, B],[A, B, A],[A, B, A, A]$, $[A, B, A, A, B]\}, L^{\prime}=[A, B],[A, B, A],[A, B, A, A],[A, B, A, A, B]$ so the dimension of $L^{\prime}$ is 4. Also, $L^{\prime \prime}=\left[L^{\prime}, L^{\prime}\right]=[A, B, A, A, B]$ so the dimension of $L^{\prime \prime}$ is 1 . Therefore, $\operatorname{dim}\left(L^{\prime} / L^{\prime \prime}\right)=3, L^{\prime \prime} \neq 0, L$ has two generators $A$ and $B$, and the structure is

Table 10.1: Basis Table for Example 1

\begin{tabular}{|c|c|c|c|c|c|c|}
\hline & $\mathbf{A}$ & $\mathbf{B}$ & {$[\mathbf{A}, \mathbf{B}]$} & {$[\mathbf{A}, \mathbf{B}, \mathbf{A}]$} & {$[\mathbf{A}, \mathbf{B}, \mathbf{A}, \mathbf{A}]$} & {$[\mathbf{A}, \mathbf{B}, \mathbf{A}, \mathbf{A}, \mathbf{B}]$} \\
\hline $\mathbf{A}$ & 0 & {$[\mathrm{~A}, \mathrm{~B}]$} & $-[\mathrm{A}, \mathrm{B}, \mathrm{A}]$ & $-[\mathrm{A}, \mathrm{B}, \mathrm{A}, \mathrm{A}]$ & 0 & 0 \\
\hline $\mathbf{B}$ & $-[\mathrm{A}, \mathrm{B}]$ & 0 & 0 & 0 & $-[\mathrm{A}, \mathrm{B}, \mathrm{A}, \mathrm{A}, \mathrm{B}]$ & 0 \\
\hline$[\mathbf{A}, \mathbf{B}]$ & {$[\mathrm{A}, \mathrm{B}, \mathrm{A}]$} & 0 & 0 & $-[\mathrm{A}, \mathrm{B}, \mathrm{A}, \mathrm{A}, \mathrm{B}]$ & 0 & 0 \\
\hline$[\mathbf{A}, \mathbf{B}, \mathbf{A}]$ & {$[\mathrm{A}, \mathrm{B}, \mathrm{A}, \mathrm{A}]$} & 0 & {$[\mathrm{~A}, \mathrm{~B}, \mathrm{~A}, \mathrm{~A}, \mathrm{~B}]$} & 0 & 0 & 0 \\
\hline$[\mathbf{A}, \mathbf{B}, \mathbf{A}, \mathbf{A}]$ & 0 & {$[\mathrm{~A}, \mathrm{~B}, \mathrm{~A}, \mathrm{~A}, \mathrm{~B}]$} & 0 & 0 & 0 & 0 \\
\hline$[\mathbf{A}, \mathbf{B}, \mathbf{A}, \mathbf{A}, \mathbf{B}]$ & 0 & 0 & 0 & 0 & 0 & 0 \\
\hline
\end{tabular}

Example 2. Let $A=E_{12}+E_{34}+E_{45}$ and $B=E_{23}+E_{56}+3 E_{35}$. Then we get

- $[A, B]=E_{13}+E_{46}-E_{36}$

- $[A, B, A]=2 E_{14}-E_{25}-E_{36}$

- $[A, B, A, A]=3 E_{15}$

- $[A, B, A, A, A]=0$

- $[A, B, B]=3 E_{15}$

- $[A, B, B, A]=0$

- $[A, B, B, B]=3 E_{16}$

- $[A, B, A, B]=0$

- $[A, B, A, A, B]=3 E_{16}$

- $[[A, B, A],[A, B]]=3 E_{16}$

Here we can see the structure is identical to that of the second class for $L\langle a, b\rangle$, when $[a, b, b]=[a, b, a, a]$. 
These last two examples are when $L=\langle a, b\rangle$. The follow examples are for the 6 isomorphism classes, when $L=\langle a, b, u\rangle$.

Example 3. Let $A=E_{12}+E_{34}+E_{45}, B=E_{23}+E_{56}$ and $U=-3 E_{26}$. Then

- $[A, B]=E_{13}+E_{46}-E_{24}$

- $[A, B, A]=2 E_{14}-E_{25}-E_{36}$

- $[A, B, A, A]=3 E_{15}$

- $[A, B, A, A, A]=0$

- $[A, B, B]=0$

- $[A, B, A, B]=0$

- $[A, B, A, A, B]=3 E_{16}$

- $[[A, B, A],[A, B]]=3 E_{16}$

- $[U, A]=3 E_{16}$

- $[U, B]=0$

We can see this is an example of case 1 of the 6 isomorphism classes, when $[u, a]=$ $z,[u, b]=0$ and $[a, b, b]=0$.

Example 4. Let $A=E_{12}+E_{34}+E_{45}, B=E_{23}+E_{56}+3 E_{35}$ and $U=-3 E_{26}$. Then

- $[A, B]=E_{13}+E_{46}-E_{36}$

- $[A, B, A]=2 E_{14}-E_{25}-E_{36}$

- $[A, B, A, A]=3 E_{15}$ 
- $[A, B, A, A, A]=0$

- $[A, B, B]=3 E_{15}$

- $[A, B, B, A]=0$

- $[A, B, B, B]=3 E_{16}$

- $[A, B, A, B]=0$

- $[A, B, A, A, B]=3 E_{16}$

- $[[A, B, A],[A, B]]=3 E_{16}$

- $[U, A]=3 E_{16}$

- $[U, B]=0$

Which is the example of case 4 , when $[u, a]=z,[u, b]=0$ and $[a, b, b]=y$.

Example 5. Let $A=E_{23}+E_{34}+E_{45}, E_{12}+E_{56}$ and $U=E_{25}$. Then

- $[A, B]=E_{46}-E_{13}$

- $[A, B, A]=-E_{14}-E_{36}$

- $[A, B, A, A]=-E_{15}+E_{26}$

- $[A, B, A, A, A]=0$

- $[A, B, B]=0$

- $[A, B, A, B]=0$

- $[A, B, A, A, B]=-2 E_{16}$

- $[[A, B, A],[A, B]]=-2 E_{16}$ 
- $[U, A]=0$

- $[U, B]=E_{26}-E_{15}$

Which we can see is an example of case 2, when $[u, a]=0,[u, b]=y$ and $[a, b, b]=0$

Example 6. If we let $A=E_{23}+E_{34}+E_{45}, E_{12}+E_{56}-\frac{1}{3} E_{24}+\frac{1}{3} E_{35}$ and $U=E_{25}$ we get an example of case 5, when $[u, a]=0,[u, b]=y$ and $[a, b, b]=y$. 


\section{Bibliography}

[1] Norman Blackburn, On a special class of p-groups, Acta Math. 100 (1958), 45-92.

[2] _ The derived group of a 2-group, Math. Proc. Cambridge Phil. Soc. 101 (1987), no. 2, 193-196.

[3] Nathan Jacobson, Basic algebra 1, 2nd ed., W. H. Freeman and Company, New York, 1985.

[4] Kailash Misra, Ma 720 lecture notes in lie algebra, 2003.

[5] Csaba Schneider, Groups of prime-power order with a small second derived quotient, Journal of Algebra 266 (2003), $539-551$. 